\title{
Analysis of small RNAs revealed differential expressions during pollen and embryo sac development in autotetraploid rice

Xiang Li ${ }^{\dagger}$, Muhammad Qasim Shahid ${ }^{\dagger}$, Juan Xia ${ }^{\dagger}$, Zijun Lu, Na Fang, Lan Wang, Jinwen Wu, Zhixiong Chen and Xiangdong Liu*

\begin{abstract}
Background: Partial pollen and embryo sac sterilities are the two main reasons for low fertility in autotetraploid rice. Our previous study revealed that small RNAs changes may associate with pollen fertility in autotetraploid rice. However, knowledge on comparative analysis between the development of pollen and embryo sac by small RNAs in autotetraploid rice is still unknown. In the present study, WE-CLSM (whole-mount eosin B-staining confocal laser scanning microscopy) and high-throughput sequencing technology was employed to examine the cytological variations and to analyze small RNAs changes during pollen and embryo sac development in autotetraploid rice compared with its diploid counterpart.
\end{abstract}

Results: A total of 321 and 368 differentially expressed miRNAs (DEM) were detected during pollen and embryo sac development in autotetraploid rice, respectively. Gene Ontology enrichment analysis on the targets of DEM associated with embryo sac and pollen development revealed 30 prominent functional gene classes, such as cell differentiation and signal transduction during embryo sac development, while only 7 prominent functional gene classes, such as flower development and transcription factor activity, were detected during pollen development in autotetraploid rice. The expression levels of $39 \mathrm{DEM}$, which revealed interaction with meiosis-related genes, showed opposite expression patterns during pollen and embryo sac development. Of these DEM, osa-miR1436_L + 3_1ss5CT and osa-miR167h-3p were associated with the female meiosis, while osa-miR159a.1 and osa-MIR159a-p5 were related with the male meiosis. 21 nt-phasiRNAs were detected during both pollen and embryo sac development, while 24 nt-phasiRNAs were found only in pollen development, which displayed down-regulation in autotetraploid compared to diploid rice and their spatial-temporal expression patterns were similar to osa-miR2275d. $24 \mathrm{nt}$ TEs-siRNAs were found to be up-regulated in embryo sac but down-regulated in pollen development.

Conclusion: The above results not only provide the small RNAs changes during four landmark stages of pollen and embryo sac development in autotetraploid rice but also have identified specifically expressed miRNAs, especially meiosis-related miRNAs, pollen-specific-24 nt-phasiRNAs and TEs-siRNAs in autotetraploid rice. Together, these findings provide a foundation for understanding the effect of polyploidy on small RNAs expression patterns during pollen and embryo sac development that may lead to different abnormalities in autotetraploid rice.

Keywords: Female gametophyte, Male gametophyte, MicroRNAs, Polyploid, phasiRNAs, TEs-siRNAs

\footnotetext{
* Correspondence: xdliu@scau.edu.cn

${ }^{\dagger}$ Equal contributors

State Key Laboratory for Conservation and Utilization of Subtropical

Agro-bioresources, South China Agricultural University, Guangzhou 510642,

China
} 


\section{Background}

Autotetraploid rice is a useful germplasm resource generated by colchicine-mediated chromosome doubling to achieve biological advantages over the diploid counterpart, such as strong hybrid vigor, higher resistance against the abiotic and biotic stresses, and the potential of the biomass production [1, 2]. However, autotetraploid rice has poor seed set, which become the biggest bottleneck and the major barrier in commercial production [3]. Partial pollen and embryo sac sterilities are the two important factors that lead to low seed set in autotetraploid rice [4-6].

Abnormal chromosome behaviors and microsporogenesis were the main cytological reasons for low pollen fertility in autotetraploid rice [6]. Moreover, we also found that abnormal microtubule organization also cause low fertility in autotetraploid rice [7]. Recently, transcriptome analysis revealed that the differential expressions of the meiosis-related or meiosis stagespecific genes may disturb the chromosome behavior during pollen development of autotetraploid rice [6]. In addition to pollen, embryo sac development is also crucial for seed set, and some abnormalities, such as embryo sac degeneration, no egg cells and abnormal position and number of polar nuclei resulted in no embryo or endosperm formation in seeds of autotetraploid rice [4]. These findings indicated that abnormal embryo sac had a strong effect on the fertility of autotetraploid rice. Recently, genome-wide gene expression profiles in ovules development have been reported and they found that dynamic gene expressions may play crucial roles during female gametogenesis in rice $[8,9]$. In addition, small RNA sequencing showed that dynamic changes in the expression patterns of miRNA and some specific differentially expressed miRNAs may cause pollen sterility in autotetraploid rice [10].

Small RNAs, the tiny size non-coding RNAs, which are 19-25 nt in length, play major roles in many biological processes, including plant reproduction [11-14]. The small RNAs can be classified into two major types, one is microRNA (miRNA), and the other is small interfering RNA (siRNA). miRNAs, as negative regulators, modulate plant gene expression by cleavage and decay of target transcripts involved in rice pollen development [15-17] and grain filling $[18,19]$. Some novel miRNAs have been reported to regulate the pollen and spikelet fertility, such as miR159, miR172, miR319 and miR397 [20-23]. The miR159, acts as the posttranscriptional factor, targeted the GAMYB-like genes (MYB33 and MYB65) and restricted the expression of these genes during anther development in Arabidopsis [20]. miR167 is essential for anther and ovule fertility in Arabidopsis, and the loss of miR167 expand the expression of ARF6 and ARF8, and cause abnormalities during ovule and anther development [24].
Furthermore, $m i R 2118$ and $m i R 2275$ are the most important miRNA families involved in the generation of male-specific 21- and 24-nt phasiRNAs (phased small-interfering RNAs), respectively, and some studies suggest that they are essential for male meiosis and pollen development $[25,26]$. siRNAs are produced from the transposable elements (TEs) and usually 24-nt in length that guide DNA methylation and histone modifications for transcriptional gene silencing. Transcriptome studies revealed that high expression of TEs have harmful effects on the Arabidopsis meiocytes [27]. Additionally, the 24 nt-siRNA abundance related to DNA methylation of class II transposable elements can suppress the expression of nearby genes in autotetraploid rice [28].

Although some information about the expression patterns of small RNAs related with pollen development are available, the expression patterns of small RNAs associated with embryo sac development in autotetraploid rice are still poorly understood. In the present study, we planned to investigate the expression levels of small RNAs during embryo sac development compared to pollen development by using an autotetraploid rice, 02428-4x and its diploid counterpart, 02428, which is a famous diploid rice variety harboring $S_{5}^{n}$ gene that can overcome the indica-japonica hybrid sterility. 02428-4x was developed from the chromosome doubling of 02428 and was self-crossed for more than 25 generations at our farm. We sought to distinguish the small RNAs changes in autotetraploid and diploid rice, and to determine the relationships between gene expression profiles and spikelet sterility.

\section{Results}

\section{Cytological observations on pollen and embryo sac} development in autotetraploid rice

The ploidy levels of autotetraploid rice, 02428-4x, was stable throughout the consecutive generations according to the chromosome observations (Additional file 1: Figure S1). We found significant variations in morphological/agronomic traits of $02428-4 x$ and $02428-2 x$ (Fig. 1; Additional file 2: Table S1). The seed set was significantly lower in $02428-4 \mathrm{x}$ than $02428-2 \mathrm{x}$, with a marked difference of $82.47 \%$. 02428-4x also depicted poor pollen fertility (43.3\%) and embryo sac fertility (55.48\%) (Additional file 2: Table S2), which may be the major reason for low seed set.

WE-CLSM (whole-mount eosin B-staining confocal laser scanning microscopy) observation revealed that the stages of pollen development in autotetraploid rice were greatly in accordance with the sampling standards of diploid rice (Additional file 2: Table S3A). The stages of pollen development were same in 02428-4x and 02428-2x, and they could be divided into eight different stages 


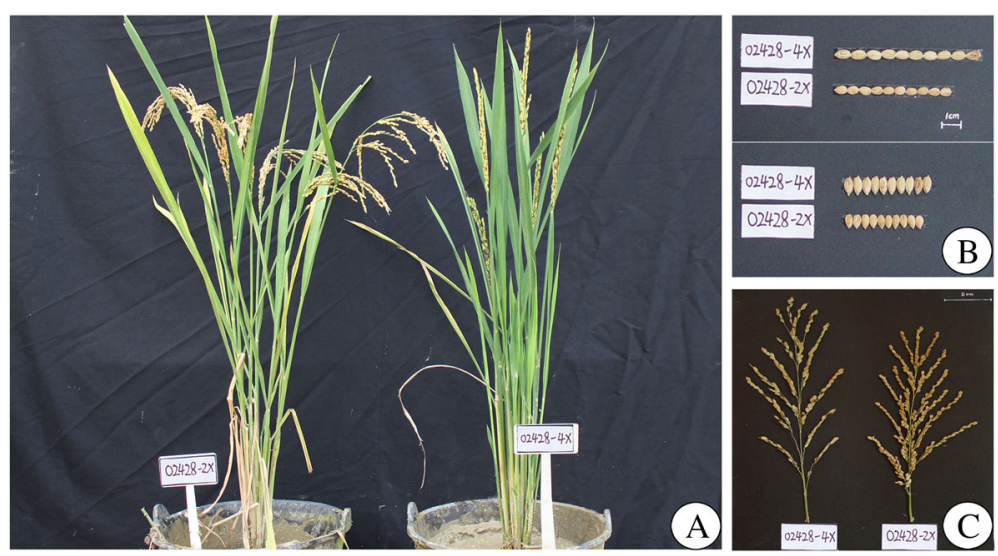

Fig. 1 Genetic variations in diploid and autotetraploid rice. (a) The diploid (left) and autotetraploid (right) rice. (b) Grain length and width of 02428-2x and 02428-4x. (c) Panicles of 02428-2x and 02428-4x

(Additional file 1: Figure S2). However, many abnormalities $(\sim 34 \%)$, including degeneration of pollen mother cell (PMC) (Fig. 2a, b, j-l), multiple nucleoli (Fig. 2c), asynchronous division of PMC (Fig. 2d), abnormal spindle in dyad (Fig. 2e), abnormal tetrads (Figs. 2f- h) and abortive pollens at bi-cellular pollen stage (Fig. 2i), were observed in 02428-4x (Additional file 2: Table S4). In addition, abnormal chromosome behavior and chromosomal configurations were found during PMC meiosis of 02428-4x (Additional file 1: Figure S3; Additional file 2: Table S5).

Moreover, WE-CLSM was also employed to observe the embryo sac development from the same spikelet. The embryo sac development stages were synchronous with the pollen development stages in diploid rice (Additional file 2: Table S3B), namely pre-meiotic interphase, meiosis, single microspore stage and bi-cellular pollen stage were corresponding to the megasporocyte formation stage, megasporocyte meiosis stage, functional megaspore formation stage and eight-nucleate embryo sac developing-stage, respectively. Similar to normal diploid rice, embryo sac development in the autotetraploid rice could be divided into eight development stages (Additional file 1: Figure S4). Different kinds of abnormalities were observed during the development of embryo sac in 02428-4x compared to 02428$2 x$. The main kinds of abnormalities were functional megaspores degeneration (Fig. 3a, b), abnormal mononucleate embryo sac (Fig. 3c), embryo sac degeneration (Fig. 3d-f), and abnormal polar nuclei in number and position (Fig. 3g, h). Some double ovules with unsynchronized development were also observed in the autotetraploid rice (Fig. 3i).

\section{Variations in miRNAs expression profiles during pollen and embryo sac development in autotetraploid rice} A total of eight miRNA libraries of four pollen stages in anther, including pre-meiotic interphase (PMA), meiosis
(MA), single microspore stage (SCP) and bi-cellular pollen stage (BCP) and four corresponding stages of embryo sac development in ovary, including megasporocyte formation stage (MF), megasporocyte meiosis stage (MM), functional megaspore formation stage (FMF) and eight-nucleate embryo sac developing-stage (EES), were prepared from autotetraploid and diploid (CK) rice. Principal component analysis showed that all libraries clustered into two groups, which related to pollen or embryo sac development, and each stage can be separated from PMA to $\mathrm{BCP} / \mathrm{MF}$ to $\mathrm{EES}$ (Additional file 1: Figure S5). Moreover, 28 miRNAs were randomly selected for $\mathrm{qPCR}$ verification (Additional file 1: Figure S6). The expression patterns of the 28 miRNAs obtained through qPCR were largely consistent with the microRNAomes data. These results demonstrated the accuracy of samples used in the present study.

A total of 748 miRNAs, including 260 known and 488 novel miRNAs, were found in the present study (Additional file 2: Table S6). Of these miRNAs, 572 and 541 expressed during the development of pollen in diploid and autotetraploid rice, while 370 and 486 were associated with the development of embryo sac in diploid and autotetraploid rice, respectively (Additional file 2: Table S7). Among the total miRNAs, 43 miRNAs (29 novel) were specifically detected during the pollen development in autotetraploid rice (12, 1, 1 and 29 in PMA- $4 \mathrm{x}, \mathrm{MA}-4 \mathrm{x}, \mathrm{SCP}-4 \mathrm{x}$ and $\mathrm{BCP}-4 \mathrm{x}$, respectively), and 49 miRNAs (47 novel) were specifically related to the development of embryo sac in autotetraploid rice (13, 20, 6 and 10 in MF-4x, MM-4x, FMF-4x and EES$4 \mathrm{x}$, respectively) (Additional file 2: Table S8).

We detected 321 DEM (Differentially expressed miRNAs) during pollen development in autotetraploid compared to diploid rice, which accounted for $\sim 50 \%$ of the total detected miRNAs. Of these DEM, 78 (i.e. 30 up- and 48 down-regulated) were detected in PMA, 

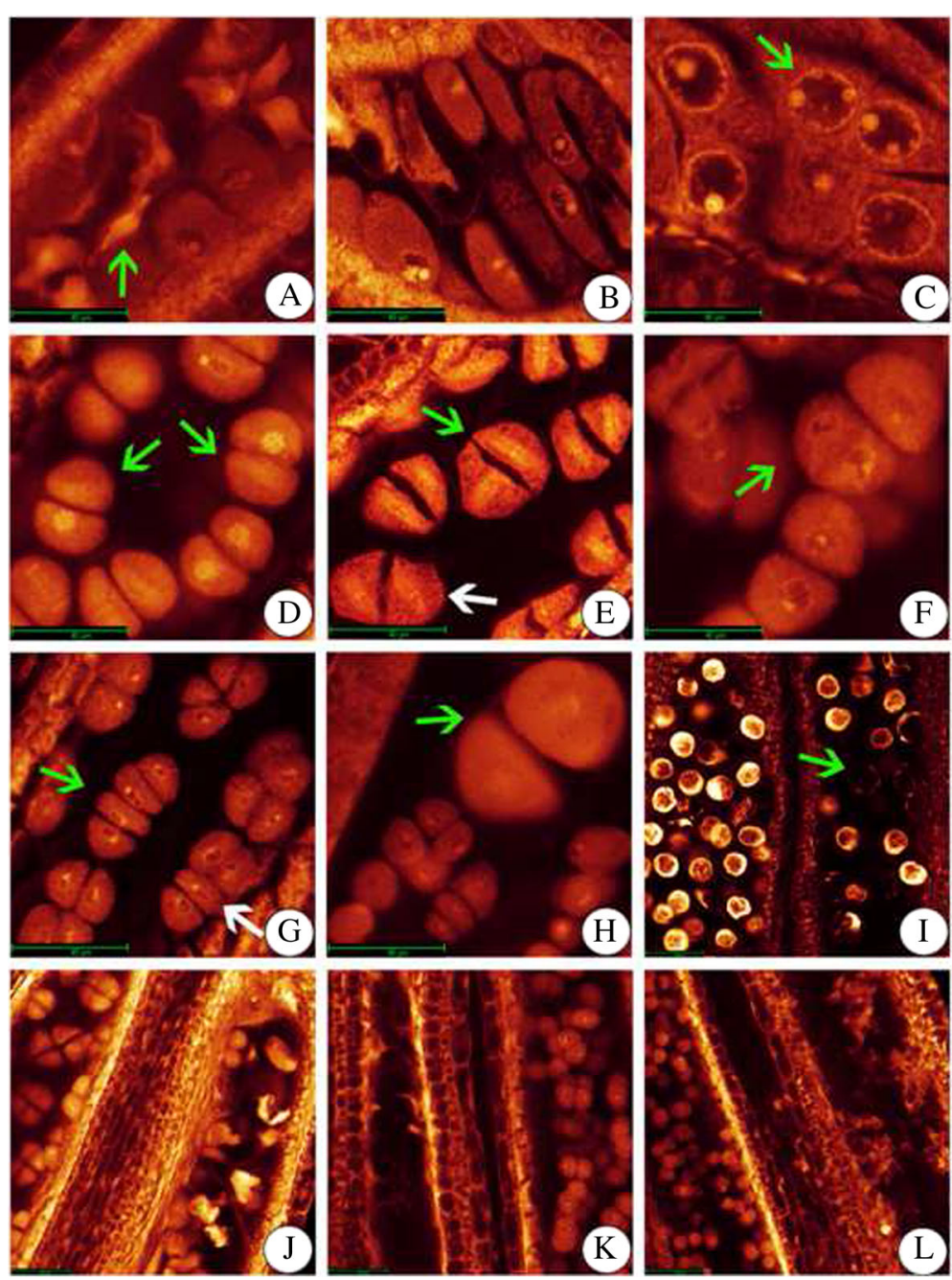

Fig. 2 Abnormalities during pollen mother cell (PMC) meiosis in 02428-4x. (a, b) degeneration of pollen mother cells (green arrow). (c) multiple nucleoli PMC (green arrow). (d) asynchronous division of PMC (green arrow), with one at metaphase II and another at prophase II. (e) abnormal spindle during formation of dyad, including 'T' type (green arrow) and oblique type (white arrow). ( $\mathbf{f}$ ) triad (green arrow). (g) abnormal tetrad, green arrow shows the linear type and white one shows the 'T' type. (h) abnormal giant tetrad (green arrow). (i) abortive pollens in bi-cellular pollen stage, with large and small pollen grains and spherical abortion type (green arrow). (j-I) degeneration of meiocytes in tetrad (j, k) and early microspore stage (I). Bars $=40 \mu \mathrm{m}$

115 (i.e. 33 up- and 82 down-regulated) in MA, 127 (i.e. 42 up- and 85 down-regulated) in SCP and 130 miRNAs (i.e. 67 up- and 63 down-regulated) in BCP of autotetraploid compared to diploid rice (Fig. 4a; Table 1). Three DEM, including PC-3p-199234_121, PC-3p-353219_42 and PC-3p-46519_401, exhibited co-down-regulation in four pollen development stages, and osa-miR2275d were found to be down-regulated in PMA, MA and SCP but up-regulated in BCP (Fig. 4b; Additional file 3: Table S9).

In total, 368 DEM were found during embryo sac development of autotetraploid rice compared to diploid rice (Fig. 4c; Table 1), including 163 (129 up- and 34 down-regulated) in MF, 252 (210 up- and 42 downregulated) in MM, 110 (68 up- and 42 down-regulated) in FMF and 65 (54 up- and 11 down-regulated) in EES. Interestingly, higher numbers of down-regulated miRNAs were detected during pollen development, while up-regulated miRNAs were more commonly found during embryo sac development. Similar phenomenon was observed by Venn analysis during embryo sac development, and the results showed that there was a great variation in number of DEM during different embryo sac development stages, and the highest number of DEM (105) was observed during MM (Fig. 4d; Additional file 3: Table S10). Venn analysis revealed that only eight miRNAs were co-up-regulated in each stage compared to diploid rice, including osa-miR1423-5p_L$2 R+2, \quad$ osa-miR1432-5p_R + 1, osa-miR1862d, osamiR2094-3p_L-1R+3, osa-miR5150-3p, osa-miR535-3p, 

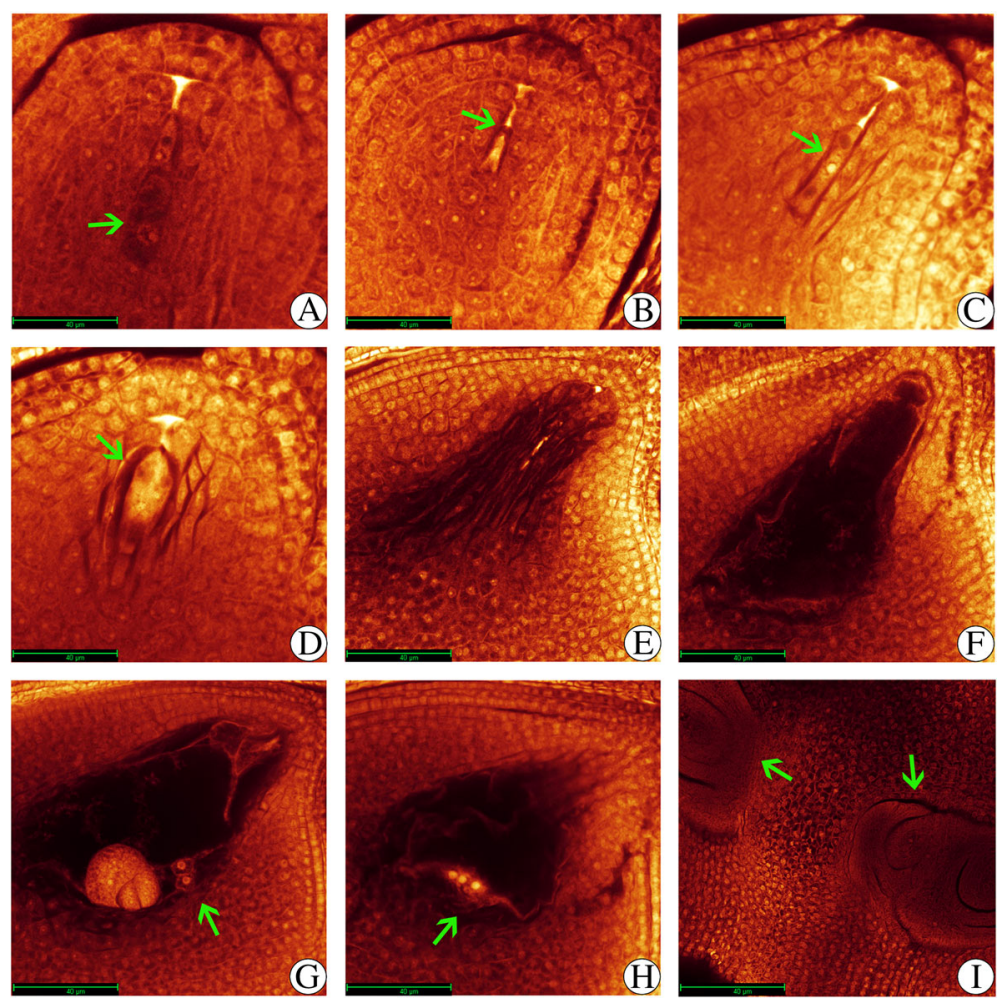

Fig. 3 Abnormalities during embryo sac development in 02428-4x. (a, b) functional megaspores degeneration (green arrow). (c) abnormal mono-nucleate embryo sac (green arrow). (d, e, f) embryo sac degeneration (green arrow). ( $\mathbf{g}, \mathbf{h}$ ) abnormal position and number of polar nuclei (green arrow). (i) double ovules (green arrow). Bars $=40 \mu \mathrm{m}$

PC-3p-115563_179 and PC-3p-46519 401 (Fig. 4d; Additional file 3: Table S10).

We further compared the DEM between pollen and embryo sac development in each corresponding stage (Additional file 1: Figure S7; Additional file 3: Table $\mathrm{S} 11)$. In the first comparison (PMA/MF), we found four DEM co-up-regulated and one DEM co-down-regulated in PMA and MF of autotetraploid rice. 18 DEM were found to be down-regulated in PMA of autotetraploid rice, but up-regulated in MF. In the second comparison (MA/MM), two DEM showed co-up-regulation and two DEM exhibited co-down-regulation during MA and MM in autotetraploid rice, respectively. 56 DEM showed opposite trends in autotetraploid rice, which were upregulated in MA but down-regulated in MM (15) or down-regulated in MA but up-regulated in MM (41). In the third comparison (SCP/FMF), nine DEM displayed co-up-regulation in SCP and FMF of autotetraploid rice, but no co-down-regulated DEM was observed. Five DEM were up-regulated in SCP but down-regulated in FMF and nine DEM were down-regulated in SCP but up-regulated in FMF. In the fourth comparison (BCP/ EES), six co-up-regulated DEM and three co-downregulated DEM were found in BCP and EES of autotetraploid rice, and six DEM showed down-regulation in
$\mathrm{BCP}$, while up-regulation in EES. Interestingly, higher number of DEM was found in each corresponding pollen and embryo sac development stage than coregulated DEM (Additional file 1: Figure S7; Additional file 3: Table S11). Furthermore, we compared the detected miRNAs between pollen and embryo sac development in autotetraploid rice. A total of $617 \mathrm{miR}$ NAs showed differential expression levels during the embryo sac development compared to pollen development (Additional file 1: Figure S8A; Additional file 3: Table S12), including 384 (i.e. 186 up- and 198 downregulated), 400 (i.e. 236 up-and 164 down-regulated), 250 (i.e. 101 up- and 149 down-regulated) and 300 DEM (i.e. 77 up- and 223 down-regulated) in MF, MM, FMF and EES, respectively. Interestingly, 111 DEM were co-expressed during all pollen and embryo sac development stages (Additional file 1: Figure S8B), including 22 co-up- and 58 co-down-regulated during embryo sac development stages in autotetraploid rice, while these DEM showed totally different expression levels during pollen development stages (i.e. 22 codown- and 58 co-up-regulated). Therefore, we defined these DEM as embryo sac-enriched miRNAs (22), which was designated as DEM-ES, and pollen-enriched miRNAs (58), which was designated as DEM-P, in 

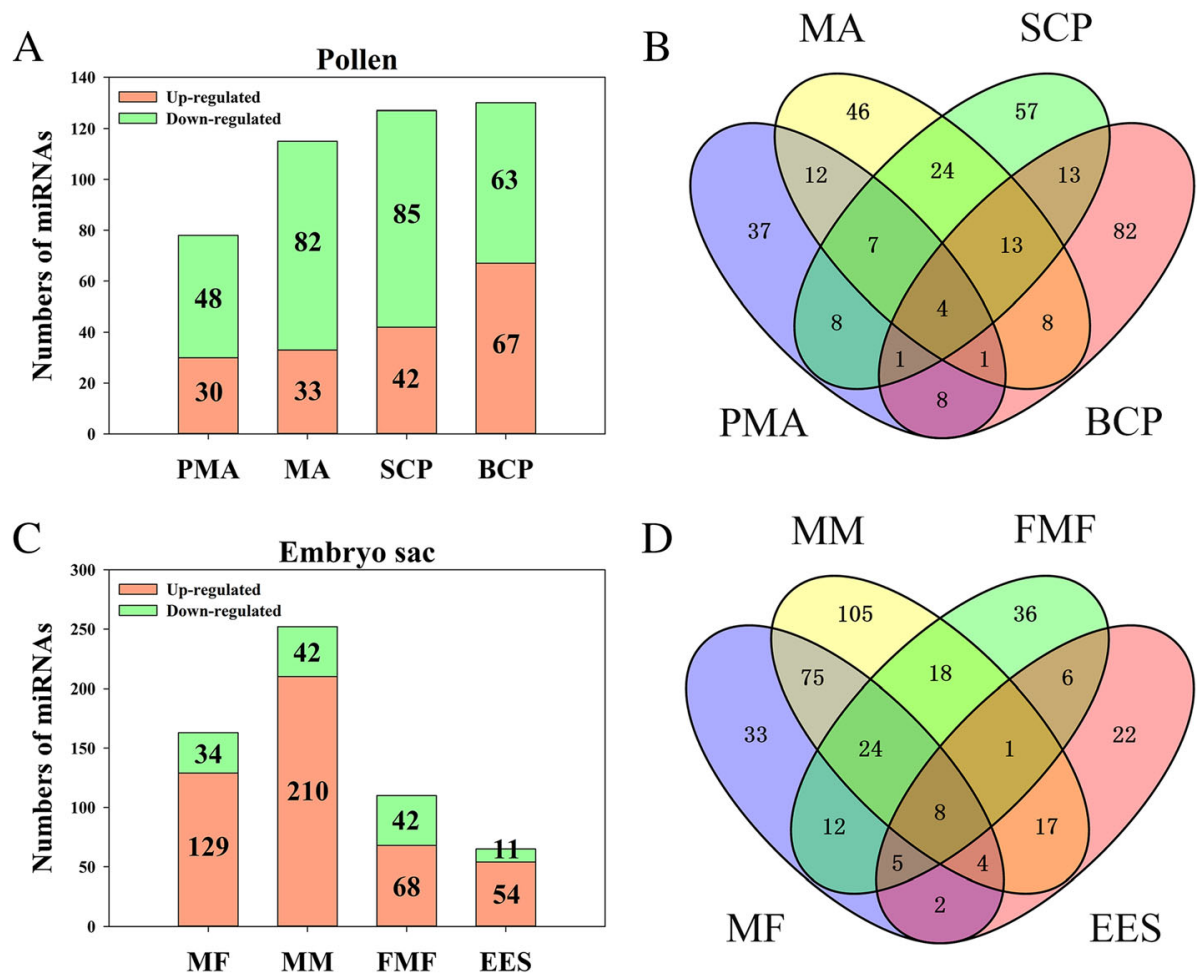

Fig. 4 Analysis of DEM (differentially expressed miRNAs) in 02428-4x compared to 02428-2x during pollen and embryo sac development. (a, c) The number of DEM at different pollen development stages (a) and embryo sac development stages (c) in 02428-4x; (b, d) Venn analysis of DEM during pollen (B) and embryo sac (d) development in 02428-4x. PMA, MA, SCP and BCP represent pre-meiotic interphase, meiosis and single microspore stage and bi-cellular pollen stage, respectively. MF, MM, FMF and EES represent megasporocyte formation stage, megasporocyte meiosis stage, functional megaspore formation stage and eight-nucleate embryo sac developing-stage, respectively

autotetraploid rice (Additional file 1: Figure S8C; Additional file 3: Table S13).

\section{Target prediction of DEM and functional classification of pollen and embryo sac mother cell meiosis-related targets}

To understand the genetic variations in autotetraploid rice during the pollen and embryo sac development, we predicted targets of 748 miRNAs using the Targetfinder, and 2260 targets were found, with an average of 3.0 targets per miRNA (Additional file 3: Table S14). Gene Ontology (GO) enrichment analyses showed that DEM$\mathrm{P}$ were significantly involved in flower development (GO: 0009908) and transcription factor activity (GO: 0003700), while cell differentiation (GO: 0030154), cell death (GO: 0008219), signal transduction (GO: 0007165) and nucleus (GO: 0005634) terms were detected by DEM-ES of autotetraploid rice (Additional file 1: Figures S9, S10). GO analysis of the targets of DEM at each stage in pollen and embryo sac development is shown in Table 1 . The predicted targets were enriched in multicellular organismal development (GO: 0007275) and extracellular region (GO: 0005576) in MA during pollen development (Additional file 1:
Figure S11). However, primary metabolic process (GO: 0044238), signal transduction (GO: 0007165), nucleotide binding (GO: 0000166), kinase activity (GO: 0016301) and transcription factor activity (GO: 0003700) were preferentially enriched in MM during embryo sac development (Additional file 1: Figure S12).

Meiosis is a crucial process during pollen development. We analyzed the DEM related to the meiosis of pollen development, and compared the target genes predicted by the DEM at MA in autotetraploid rice with the transcriptome data reported for meiosis-related and stage-specific gene expressions in Arabidopsis, diploid and autotetraploid rice [6, 29-37]. By using the predicted targets of the up-regulated miRNAs in MA, we identified 18 DEM associated with meiosis (Additional file 3: Table S15). Two meiosis-related genes (i.e. LOC_Os02g48010 predicted by osa-MIR5083-p3 that encoded nuclear matrix constituent protein 1-like and $L O C_{-} O s 02 g 42230$ predicted by osaMIR159a-p5 that encoded RPA2B - Putative singlestranded DNA binding complex subunit 2), and three meiosis specific genes that showed down-regulation in autotetraploid compared to the diploid rice during meiosis, including LOC_Os09g32020 (predicted by osa-MIR159ap5), LOC_Os06g40550 (predicted by osa-miR5504_R-3), 
Table 1 Differentially expressed miRNAs (DEM) in autotetraploid rice compared to diploid rice

\begin{tabular}{|c|c|c|c|c|c|}
\hline Comparison & Up-regulated & Down-regulated & Total & Targets & Gene Ontology \\
\hline PMA-4x/2x & 30 & 48 & 78 & 470 & GO: 0005576 (extracellular region) \\
\hline$M A-4 x / 2 x$ & 33 & 82 & 115 & 664 & $\begin{array}{l}\text { GO: } 0007275 \text { (multicellular organismal development) } \\
\text { GO: } 0005576 \text { (extracellular region) }\end{array}$ \\
\hline SCP- $4 x / 2 x$ & 42 & 85 & 127 & 504 & $\begin{array}{l}\text { GO: } 0006139 \text { (nucleobase, nucleoside, nucleotide and } \\
\text { nucleic acid metabolic process) } \\
\text { GO: } 0009653 \text { (anatomical structure morphogenesis) } \\
\text { GO: } 0030154 \text { (cell differentiation) } \\
\text { GO: } 0007165 \text { (signal transduction) } \\
\text { GO: } 0009908 \text { (flower development) } \\
\text { GO: } 0003700 \text { (transcription factor activity) }\end{array}$ \\
\hline$B C P-4 x / 2 x$ & 67 & 63 & 130 & 548 & $\begin{array}{l}\text { GO: } 0009908 \text { (flower development) } \\
\text { GO: } 0007165 \text { (signal transduction) } \\
\text { GO: } 0009653 \text { (anatomical structure morphogenesis) } \\
\text { GO: } 0006139 \text { (nucleobase, nucleoside, nucleotide and } \\
\text { nucleic acid metabolic process) } \\
\text { GO: } 0003700 \text { (transcription factor activity) } \\
\text { GO: } 0003723 \text { (RNA binding) }\end{array}$ \\
\hline$M F-4 x / 2 x$ & 129 & 34 & 163 & 489 & $\begin{array}{l}\text { GO: } 0009908 \text { (flower development) } \\
\text { GO: } 0009653 \text { (anatomical structure morphogenesis) } \\
\text { GO: } 0006139 \text { (nucleobase, nucleoside, nucleotide and } \\
\text { nucleic acid metabolic process) } \\
\text { GO: } 0007165 \text { (signal transduction) } \\
\text { GO: } 0030154 \text { (cell differentiation) } \\
\text { GO: } 0003700 \text { (transcription factor activity) } \\
\text { GO: } 0016301 \text { (kinase activity) }\end{array}$ \\
\hline$M M-4 x / 2 x$ & 210 & 42 & 252 & 835 & $\begin{array}{l}\text { GO: } 0044238 \text { (primary metabolic process) } \\
\text { GO: } 0007165 \text { (signal transduction) } \\
\text { GO: } 0000166 \text { (nucleotide binding) } \\
\text { GO: } 0016301 \text { (kinase activity) } \\
\text { GO: } 0003700 \text { (transcription factor activity) }\end{array}$ \\
\hline FMF- $4 x / 2 x$ & 68 & 42 & 110 & 432 & $\begin{array}{l}\text { GO: } 0009856 \text { (pollination) } \\
\text { GO: } 0009653 \text { (anatomical structure morphogenesis) } \\
\text { GO: } 0005488 \text { (binding) }\end{array}$ \\
\hline $\mathrm{EES}-4 \mathrm{x} / 2 \mathrm{x}$ & 54 & 11 & 65 & 285 & GO: 0003700 (transcription factor activity) \\
\hline
\end{tabular}

PMA, MA, SCP and BCP represent pre-meiotic interphase, meiosis and single microspore stage and bi-cellular pollen stage, respectively. MF, MM, FMF and EES represent megasporocyte formation stage, megasporocyte meiosis stage, functional megaspore formation stage and eight-nucleate embryo sac developing-stage, respectively. " $4 \mathrm{x}$ " and " $2 \mathrm{x}$ " represent the autotetraploid and diploid rice, respectively

and LOC_Os04g21590 (predicted by osa-miR5504_R-3), which annotated as ubiquitin fusion degradation protein, ABC-2 type transporter domain containing protein and PB1 domain containing protein, respectively. Another important gene, LOC_Os01g59660 (OsGAMYB, predicted by osa-miR159a.1), which is required for pollen development and encodes MYB family transcription factor, was also detected. Some important genes, such as LOC_Os07g36940 predicted by the osa-miR5836, and LOC_Os01g47530 predicted by the osa-miR159a.1, were found to be downregulated during meiosis stage (Additional file 3: Table S15). Moreover, 14 genes showed differential expression patterns in transcriptome sequencing, and the targets of up-regulated DEM were similar to these 14 genes, such as LOC_Os03g63390 (predicted by osa-miR528-5p) annotated as plastocyanin-like domain containing protein, $L O C \_O s 08 g 23880$ (predicted by gma-miR6300_R + 3) annotated as no apical meristem (NAM) protein and LOC_Os10g30150 (predicted by osa-miR3979-5p_R +1 ) annotated as universal stress protein domain containing protein (Additional file 3: Table S15). All the aforementioned targets may relate to abnormal meiosis of PMC.

The development process of embryo sac was different from pollen, especially after meiosis of the embryo sac mother cell (EMC); therefore, the regulation mechanism of embryo sac development was also different from that in pollen. We compared the target genes predicted by the DEM in MM associated with the embryo sac development in autotetraploid rice with the previously reported transcriptome data for megagametogenesisrelated and stage-specific expression in diploid rice and Arabidopsis [8, 9, 38-40], and 21 DEM were found to be related to the development of embryo sac mother cell (Additional file 3: Table S16). Among the up-regulated miRNAs of MM in autotetraploid, 26 predicted targets were associated with the megasporocyte meiosis stage. Two genes, LOC_Os06g47830 (RPA2C, predicted by osamiR167h-3p) and LOC_Os12g20324 (CYCA1;3, predicted by $\left.P C-5 p-896126 \_17\right)$ were associated with meiosis. LOC_Os06g11500 and LOC_Os06g48060 (predicted by 
osa-miR1436_L + 3_1ss5CT, a specific DEM in MM), annotated as minichromosome maintenance (MCM) family subunit 9 and $\mathrm{ABC}$ transporter, were highly expressed in zygotene and pachytene of EMC. Nine genes showed the highest expression in tetrad, such as LOC_Os12g42160, which encoded kinesin motor domain containing protein (predicted by the MM specific DEM, PC-5p-983436_13 and osa-miR1436_L +3_1ss5CT) and LOC_Os06g48030 (predicted by the MM specific DEM, osa-MIR814ap3_1ss24AT and PC-5p-430417_41) (Additional file 3: Table S16).

Moreover, we used the targets of the DEM in MA and $\mathrm{MM}$ to analyze protein-protein interaction networks. Of these targets, eight genes associated with meiosis exhibited interactions with 39 targets, including LOC_Os09g32020 (OsTKPR1, ubiquitin fusion degradation protein), LOC_Os08g40440 (dihydroflavonol-4-reductase) and LOC_Os06g40550 (ABC-2 type transporter domain containing protein), identified by Fujita et al. [29], LOC_Os05g51790 (SMC5, RecF/RecN/SMC N terminal domain containing protein) and LOC_OsO2g42230 (RPA2B - Putative single-stranded DNA binding complex subunit 2) identified by Jin et al. [31], LOC_Os01g59660 (OsGAMYB, MYB family transcription factor) identified by Aya et al. [32], LOC_Os06g47830 (RPA2C - Putative single-stranded DNA binding complex subunit 2) identified by Wang et al. [40] and LOC_Os12g20324 (CYCA1;3) found in oryzabase (http://www.shigen.nig.ac.jp/rice/oryzabase/) (Additional file 1: Figure S13; Additional file 4: Table S17). The DEM detected in MA during pollen development was different from the embryo sac development, and these DEM showed divergent expression patterns. OsGAMYB encoded by LOC_Os01g59660 (predicted by osa-miR159a.1, which showed up-regulation in male meiosis but down-regulation in female meiosis), revealed interaction with the genes encoding splicing factor 3B subunit 1 ( $L O C_{-} O s 02 g 05410$ and $L O C_{-}$ Os02g05310 predicted by osa-miR5504_R-3). CYCA $1 ; 3$ (LOC_Os12g20324 predicted by PC-5p-896126_17, which showed up-regulation in female meiosis) interacted with the genes encoding MYB family transcription factor and putative minichromosome maintenance (MCM) family subunit 9 ( LOC_Os06g11500 predicted by osa-miR1436_L + 3_1ss5CT, which displayed downregulation in male meiosis but up-regulation in female meiosis). RPA2C (LOC_Os06g47830 predicted by osamiR167h-3p) and RPA2B (LOC_OsO2g42230 predicted by osa-MIR159a-p5, which found to be up-regulated in male meiosis but down-regulated in female meiosis) encoded replication proteins, $\mathrm{A} 2 \mathrm{C}$ and $\mathrm{A} 2 \mathrm{~B}$, and showed interaction with $L O C_{-} O s 06 g 11500$, which further interacted with CYCA1;3. OsTKPR1 (LOC_Os09g32020 encoded ubiquitin fusion degradation protein), which was another target predicted by osa-MIR159a-p5, interacted with the mov34 family protein (LOC_Os05g46490 predicted by osa-miR1436_L +3_1ss5CT), Sel1 repeat domain containing protein (LOC_OsO3g15350 predicted by osa-miR1436_L+3_1ss5CT) and DnaK family protein genes (LOC_Os12g14070, predicted by osamiR3979-5p_R + 1, which showed up-regulation in male meiosis, and LOC_Os02g53420 and LOC_OsO3g02260, predicted by $o s a-m i R 164 e \_R-3$, which displayed downregulation in female meiosis). The SMC5 gene encoded RecF/RecN/SMC N terminal domain containing protein (LOC_Os05g51790 predicted by osa-miR1861h), which interacted with LOC_Os05g05440 (annotated as expressed protein and predicted by osa-MIR5083- 33 , which was found to be up-regulated in male meiosis but down-regulated in female meiosis) and LOC_OsO8g37444 (annotated as signal recognition particle receptor and predicted by hvu-miR1436_L +3_2ss5CT13GA, which exhibited up-regulation in female meiosis). In addition, LOC_Os08g40440 (predicted by osa-miR1436_L+ 3_1ss5CT) encoded dihydroflavonol-4-reductase that interacted with $\mathrm{ABC}-2$ type transporter domain containing protein (LOC_OsO6g40550 predicted by osa-miR164e_R-3, osa-miR5504_R-3) and FG-GAP repeat-containing protein (LOC_Os03g61050 predicted by osa-miR812v_1ss4GA that up-regulated in female meiosis). Overall, complex proteinprotein interaction networks were detected by the meiosisrelated gene and meiosis-related DEM.

\section{PhasiRNAs accumulation during the pollen and embryo sac development in autotetraploid rice}

The expression patterns of $m i R 2118$ and $m i R 2275$ families from PMA to BCP in pollen and from MF to EES in embryo sac were nearly consistent in 02428-4x and 02428-2x, which specifically enriched during pollen development and gradually decreased after PMA (Fig. 5). Six members of miR2118 family showed differential expression patterns during pollen development (Table 2). Two of them, osa-miR2118d and osa-MIR2118e-p5, were found to be up-regulated in MA. Beside, six members of miR2275 also exhibited differential expression patterns in 02428-4x, including osa-miR2275d, osa-MIR2275c-p3, mes-miR2275, $z m a-m i R 2275 a-3 p \_L-1, \quad z m a-m i R 2275 b-3 p \_1 s s 10 C T$ and $z m a-m i R 2275 b-3 p \_1 s s 22 A G$ (Table 2). Interestingly, all these six miRNAs of miR2275 family were found to be down-regulated in MA of 02428-4x.

Based on PhaseTank analysis [41], we observed 1117 loci generating $21 \mathrm{nt}$-phasiRNAs and 190 loci generating 24 nt-phasiRNAs triggered by the miR2118 and miR2275, respectively (Additional file 4: Tables S18, S19). We found that $21 \mathrm{nt}$-phasiRNAs were not only detected during the development of pollen, but also during the development of embryo sac, while 24 nt-phasiRNAs only accumulated during the pollen development (Figs. 6a, b). By Z-score transformation [34], 45121 nt- 

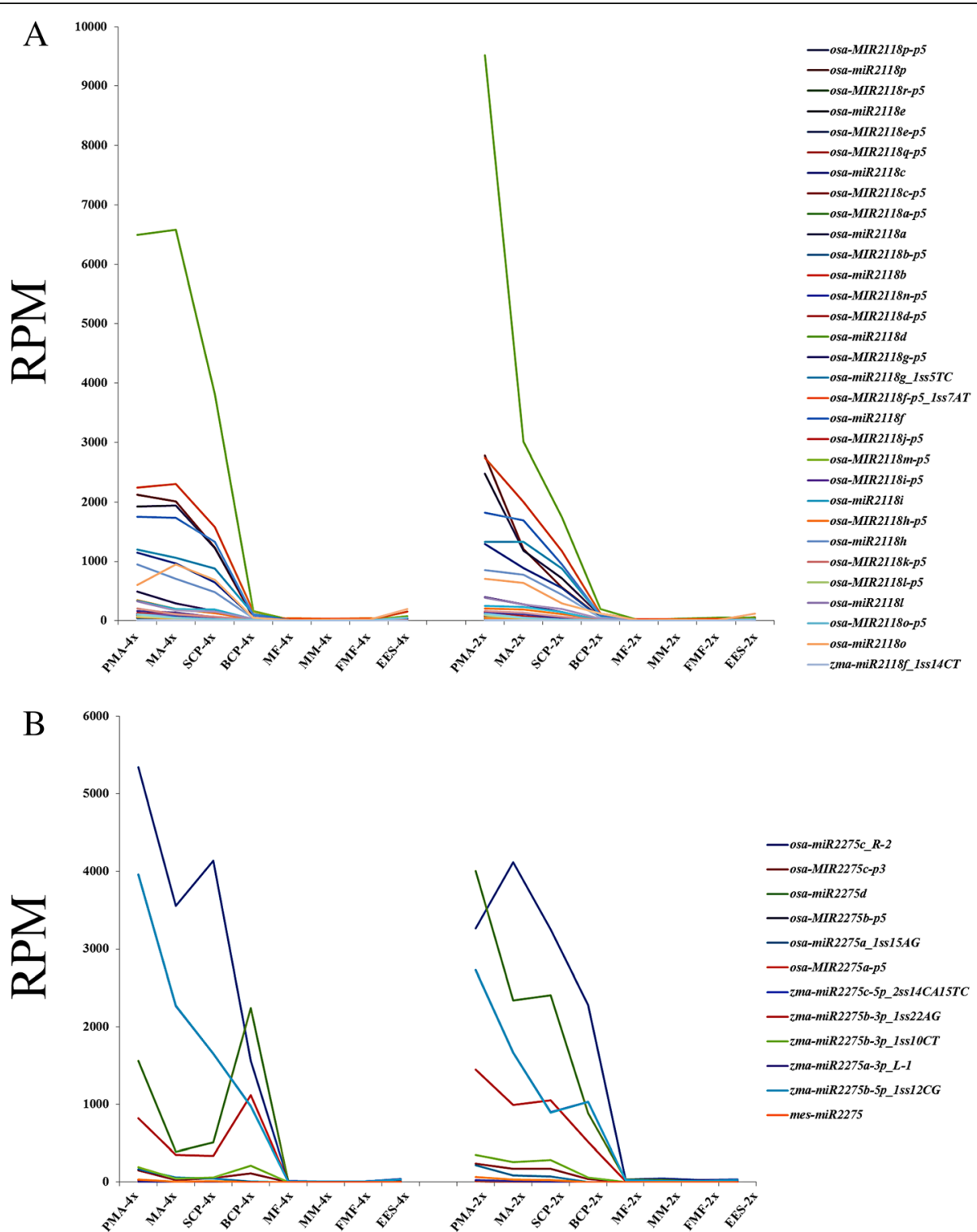

Fig. 5 The expression patterns of miR2118 (a) and miR2275 (b) families between 02428-4x and 02428-2x. PMA, MA, SCP and BCP represent pre-meiotic interphase, meiosis and single microspore stage and bi-cellular pollen stage, respectively. MF, MM, FMF and EES represent megasporocyte formation stage, megasporocyte meiosis stage, functional megaspore formation stage and eight-nucleate embryo sac developing-stage, respectively. " $4 x$ " and "2x" represent the autotetraploid and diploid rice, respectively

phasiRNAs and 17924 nt-phasiRNAs were preferentially expressed during the pollen development. Pollenspecific-21 nt-phasiRNAs were accumulated from PMA and retained the expression levels in $\mathrm{BCP}$ of $02428-2 \mathrm{x}$, and similar trend was detected in 02428-4x (Fig. 6c). In contrast, pollen-specific-24 nt-phasiRNAs appeared at PMA and reached to the highest level in SCP, and then declined in BCP of 02428-2x, whereas gradual decline was observed in 02428-4x (Fig. 6d). Furthermore, only 109 out of 451 pollen-specific-21 nt-phasiRNAs showed different levels of expression between 02428-4x and 02428-2x. Upregulated pollen-specific- $21 \mathrm{nt}$-phasiRNAs were dominant in PMA and BCP, but more number of down-regulated pollen-specific-21 nt-phasiRNAs was detected in MA and SCP (Table 3). The expression levels of miR2118 (i.e. osamiR2118d, osa-miR2118p, osa-miR2118o, osa-miR2118i) and their pollen-specific-21 nt-phasiRNAs were quite different during pollen development stages (Tables 2 and 3). A total of 173 out of 179 pollen-specific-24 nt-phasiRNAs showed differential expression patterns in 02428-4x compared to $02428-2 x$. In addition, almost all of the differentially expressed pollen-specific-24 nt-phasiRNAs were found to be down-regulated during pollen development in autotetraploid rice, especially at MA and SCP, which were 
Table 2 The regulation of miR2118 and miR2275 families during pollen development of autotetraploid rice

\begin{tabular}{|c|c|c|c|c|c|}
\hline miRNA Family & miRNA name & PMA & MA & SCP & $\mathrm{BCP}$ \\
\hline \multirow[t]{6}{*}{ miR2118 } & osa-miR2118d & & up & up & \\
\hline & osa-miR2118p & & & up & \\
\hline & osa-miR2118o & & & up & down \\
\hline & osa-miR2118i & & & & up \\
\hline & osa-MIR2118i-p5 & & & up & \\
\hline & osa-MIR2118e-p5 & & up & & \\
\hline \multirow[t]{6}{*}{$\operatorname{miR} 2275$} & osa-miR2275d & down & down & down & up \\
\hline & mes-miR2275 & down & down & down & \\
\hline & osa-MIR2275c-p3 & & down & down & up \\
\hline & zma-miR2275b-3p_1ss10CT & & down & down & up \\
\hline & zma-miR2275b-3p_1ss22AG & & down & down & up \\
\hline & zma-miR2275a-3p_L-1 & down & down & & \\
\hline
\end{tabular}

Up: up-regulated in autotetraploid rice; Down: down-regulated in autotetraploid rice; PMA, MA, SCP and BCP represent pre-meiotic interphase, meiosis and single microspore stage and bi-cellular pollen stage, respectively

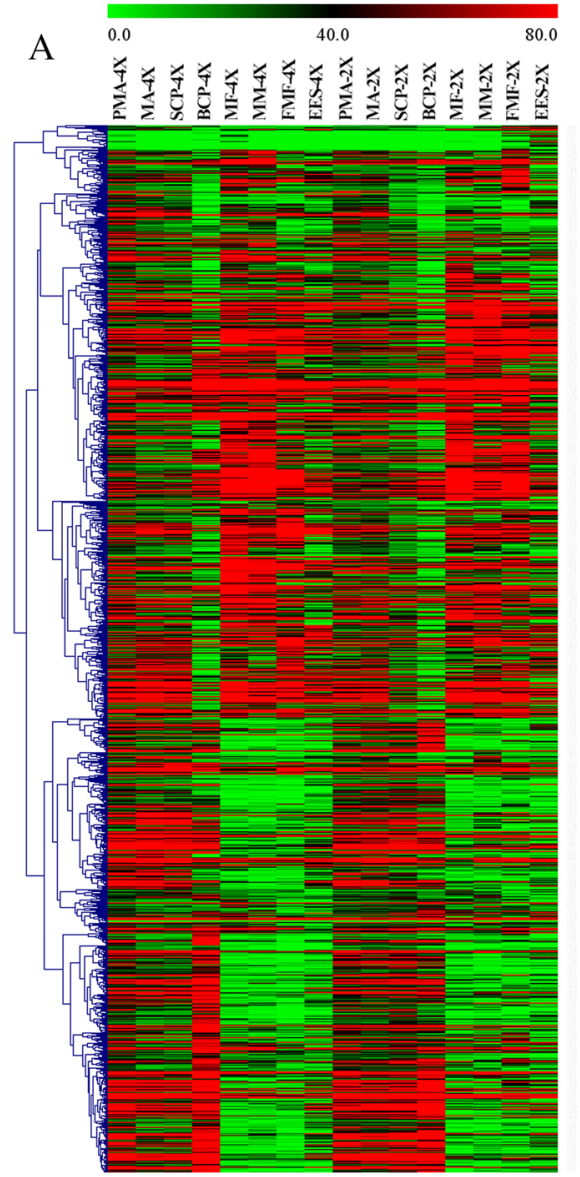

B
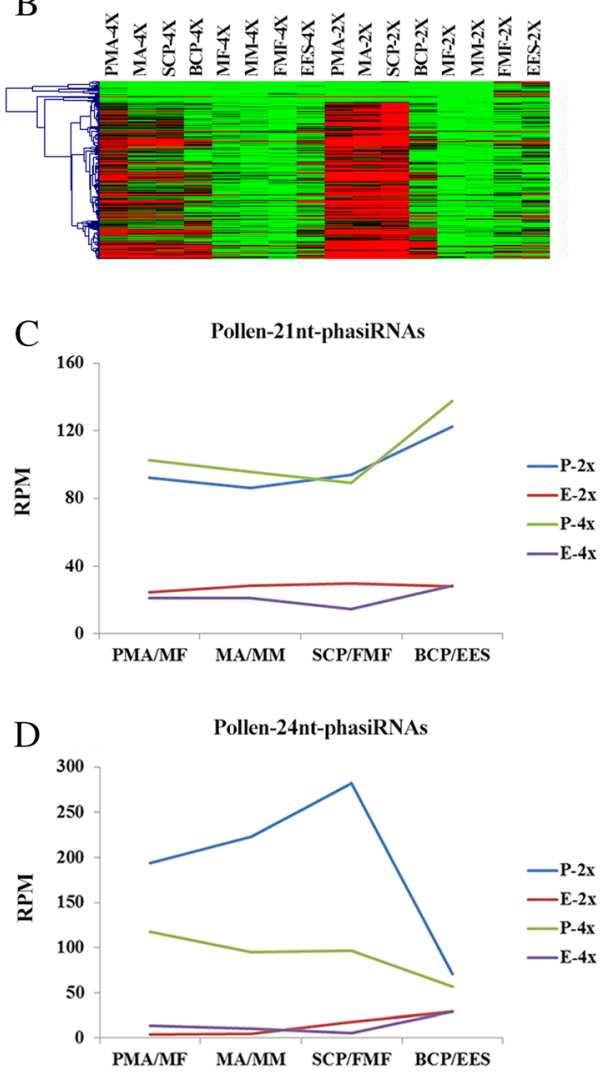

Fig. 6 The distribution of 21 nt- and 24-phasiRNAs in auotetraploid and diploid rice. (a, b) Hierarchical cluster analysis of 21 nt-phasiRNAs (a) and 24-phasiRNAs (b) in different libraries was constructed by MultiExperiment View (version 4.9). The scale bar indicates the RPM of phasiRNAs; (c, d) Abundance of $21 \mathrm{nt}$-phasiRNAs and $24 \mathrm{nt}$-phasiRNAs in different libraries. P-4x and P-2x indicate autotetraploid and diploid pollen development. $\mathrm{E}-4 \mathrm{x}$ and $\mathrm{E}-2 \mathrm{x}$ indicate autotetraploid and diploid embryo sac development, respectively 
Table 3 Differentially expressed phasiRNAs during pollen development in autotetraploid rice

\begin{tabular}{|c|c|c|c|c|c|c|c|c|}
\hline & \multicolumn{2}{|c|}{ PMA } & \multicolumn{2}{|l|}{ MA } & \multicolumn{2}{|c|}{ SCP } & \multicolumn{2}{|c|}{ BCP } \\
\hline & Up & Down & Up & Down & Up & Down & Up & Down \\
\hline 21 nt-phasiRNAs & 18 & 2 & 30 & 50 & 35 & 98 & 79 & 22 \\
\hline osa-miR2118d & 4 & 0 & 6 & 20 & 5 & 25 & 23 & 5 \\
\hline osa-miR2118p & 4 & 0 & 6 & 4 & 6 & 11 & 13 & 1 \\
\hline osa-miR2118o & 6 & 0 & 7 & 12 & 7 & 26 & 21 & 5 \\
\hline osa-miR2118i & 1 & 0 & 1 & 2 & 1 & 8 & 5 & 2 \\
\hline 24 nt-phasiRNAs & 6 & 38 & 0 & 138 & 0 & 153 & 22 & 13 \\
\hline osa-miR2275d & 2 & 14 & 0 & 54 & 0 & 63 & 9 & 5 \\
\hline
\end{tabular}

Up: up-regulated in autotetraploid rice; Down: down-regulated in autotetraploid rice; $\mathrm{PMA}, \mathrm{MA}, \mathrm{SCP}$ and $\mathrm{BCP}$ represent pre-meiotic interphase, meiosis and single microspore stage and bi-cellular pollen stage, respectively. osa-miR2118d, osamiR2118p, osa-miR2118o and osa-miR2118i indicate the 21 nt-phasiRNAs triggered by them; osa-miR2275d indicates the $24 \mathrm{nt}$-phasiRNAs triggered by it

in accordance with the spatial-temporal expressions of $m i R 2275$ (i.e. osa-miR2275d, which was down-regulated from PMA to SCP and up-regulated in BCP) (Tables 2 and 3).

\section{siRNAs, associated with transposable elements, showed} differential expression patterns in autotetraploid rice We documented 4386 siRNAs associated with transposable elements (TEs-siRNAs) during pollen and embryo sac development of 02428-4x and 02428-2x. 24-nt TEs-siRNAs, 3106 of 4386 siRNAs, occupied the great majority of the siRNA population (Additional file 4: Table S20). Of 3106 TEs-siRNAs (609 associated with pollen development library and 2734 associated with embryo sac development library), 372 and 2497 were specifically associated with pollen and embryo sac development, respectively, and 237 TEs-siRNAs were expressed during both pollen and embryo sac development (Additional file 1: Figure S14). In contrast to the pollen development, the $24 \mathrm{nt}$ TEs-siRNAs were abundant in embryo sac development. We classified these $24 \mathrm{nt}$ TEs-siRNAs in class I (retrotransposons) with five types and class II (transposon) with eight types (Additional file 4: Table S21). 24 nt TEs-siRNAs showed different expression patterns during the pollen and embryo sac development in $02428-4 x$, as well as in the $02428-2 x$; however, some changes were found during the pollen and embryo sac development when compared to each other (Fig. 7). Compared to the diploid rice, 24 nt TEs-siRNAs were abundantly expressed during embryo sac development of 02428-4x, whereas low levels of expressions were found in pollen development of 02428-4x (Fig. 7). Of these $24 \mathrm{nt}$ TEs-siRNAs, 59\% (584/988) of MF, 69\% (1152/1672) of MM, 49\% (524/1066) of FMF and 25\% (475/1877) of EES were differentially expressed during embryo sac development of $02428-4 x$, and 39\% (113/287) of PMA, 50\% (148/
296) of MA, 57\% (179/312) of SCP and 56\% (115/206) of BCP were differentially expressed during pollen development (Additional file 4: Table S22). Most of the differentially expressed TEs-siRNAs (96\%, 96\%, 92\% and 85\% in MF, MM, FMF and EES, respectively) were up-regulated in embryo sac development of 02428-4x. Particularly, the MM stage exhibited the highest numbers (1106) of differentially expressed TEs-siRNAs. On the other hand, higher number of down-regulated TEs-siRNAs (81\%, 84\%, 79\% and $88 \%$ in PMA, MA, SCP and BCP) were identified in 02428-4x than 20428-2x during the pollen development (Additional file 4: Table S22).

\section{Discussion}

Polyploidy associated miRNAs expression patterns during pollen and embryo sac development in autotetraploid rice Transcriptome analysis of rice ovules and anthers revealed that only 448 genes representing ovule-exclusive genes, compared to 1149 anther-exclusive genes, and different GO terms were found in ovules and anthers [8]. Here, large numbers of miRNAs were detected in pollen and embryo sac library and spatiotemporally dynamics during the pollen and embryo sac development. We found some miRNAs were exclusive during the development of pollen or embryo sac and some were exclusive in autotetraploid/ diploid rice; suggesting that miRNAs expressed during pollen development were different from embryo sac development, and some miRNAs changed after polyploidization. MircoRNAome analysis of different species of diploid and autotetraploid Paulownia showed that upregulated miRNAs were more common than down-regulated miRNAs in autotetraploid P. tomentosa, P. fortunei, and $P$. australis except in autotetraploid P. 'Yuza 1' [4245]. In our study, the number of down-regulated DEM was higher in pollen development of autotetraploid than diploid rice, whereas Li et al. [10] reported higher number of up-regulated DEM in pollen development of another autotetraploid line than diploid counterpart. Beside, differences between the pollen and embryo sac development in 02428-4x, up-regulated DEM were enriched during embryo sac development, particularly at the meiosis stage. These studies on polyploid plants suggest greater diversity in miRNAs expression patterns in different species. Polyploidization always cause increase/ decrease in expression patterns, gene loss and gene recombination in many plant species [46-49]. Higher missing rate of markers were detected in Alfalfa [50], great variations were found in miRNAs [51, 52], and some differentially expressed miRNAs were found to be associated with different ploidy rice [53]. Here, multitudinous of exclusively DEM detected in each stage during pollen and embryo sac development in autotetraploid rice, and similar phenomena was found in Taichung65-4x [10], where miRNA expression patterns changed 

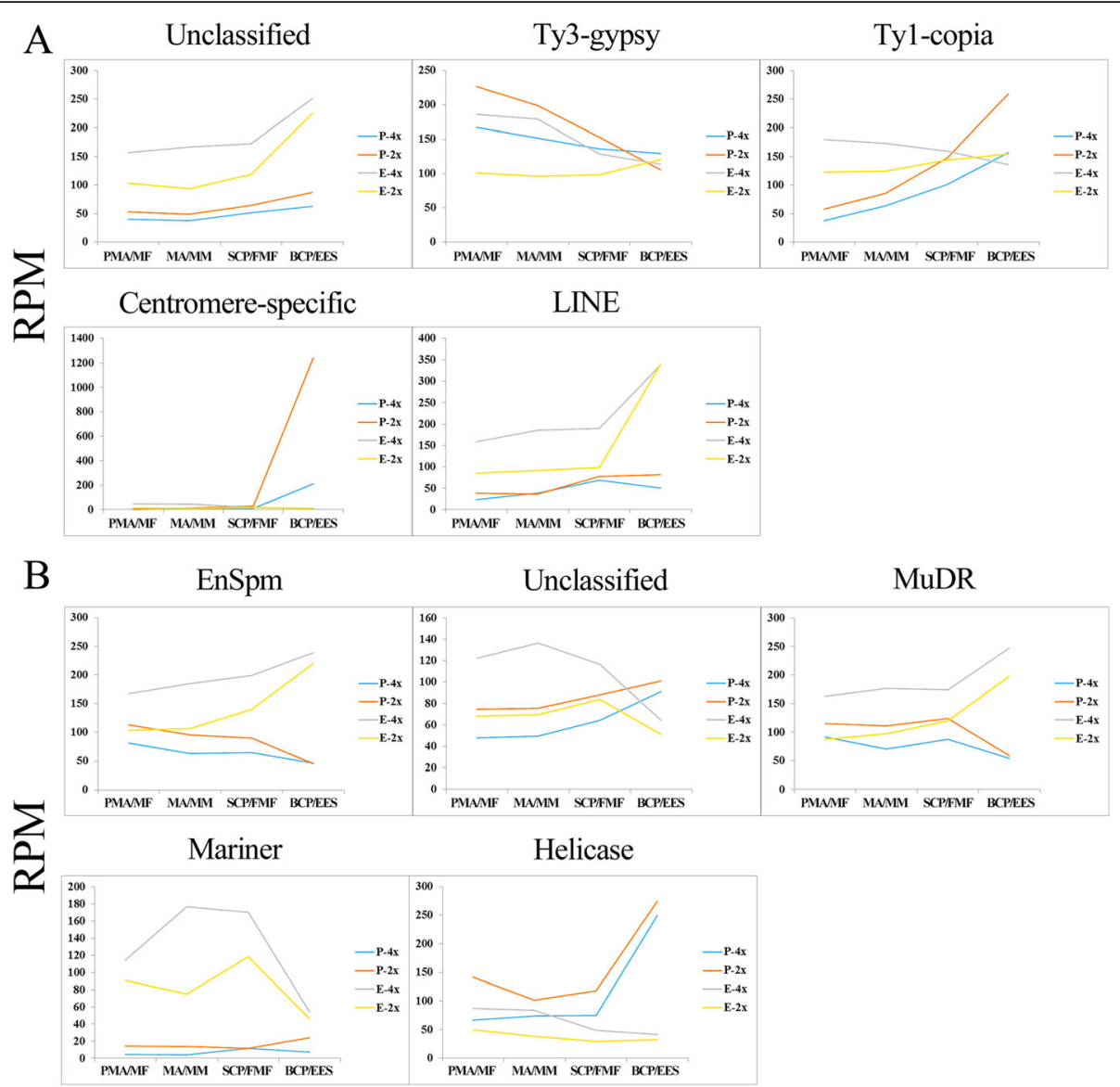

Fig. 7 Abundance of TEs-siRNAs (siRNAs associated with transposable elements) in 02428-4x and 02428-2x during pollen and embryo sac development. (a) 24 nt TEs-siRNAs in class I (retrotransposons). (b) 24 nt TEs-siRNAs in class II (transposon). PMA, MA, SCP and BCP represent pre-meiotic interphase, meiosis and single microspore stage and bi-cellular pollen stage, respectively. MF, MM, FMF and EES represent megasporocyte formation stage, megasporocyte meiosis stage, functional megaspore formation stage and eight-nucleate embryo sac developing-stage. P-4x and P-2x indicate autotetraploid and diploid pollen development, respectively. E-4x and E-2x indicate autotetraploid and diploid embryo sac development, respectively

dynamically in autotetraploid rice. These studies demonstrated huge changes in miRNAs after polyploidization and polyploidy displayed the major impact on the miRNAs expression pattern. Taken together, a great diversity in the DEM was exclusively documented in autotetraploid rice, and some co-expressed DEM exhibited ploidyrelated expression patterns, such as osa-miR2275d, probably played a key role, which may be associated with the pollen and embryo sac sterility in autotetraploid rice.

\section{The contrasting expression patterns of meiosis-related DEM during pollen and embryo sac development in autotetraploid rice}

Transcriptome profiling is helpful tool for investigating the molecular characters of male and female gametophytes development in Arabidopsis [35, 39]. Genome-wide gene expression profiles of ovules and anthers revealed that dynamic gene expressions play crucial roles during female and male gametogenesis in rice $[8,9,29,30]$. In the present work, the anthers/ovaries containing the developing male/ female gametocytes at specific stages were observed, and identified the targets of DEM associated with meiosis, which were similar to the genes detected by previous studies [8, 9, 29-33]. A total of 18 and 21 DEM associated with meiosis were detected in male and female gametophytes, respectively, and these DEM showed huge differences between male and female meiosis, and probably played important roles during pollen and embryo sac development in autotetraploid rice. The rest of the DEM might be involved in various distinct processes that may also related to reproductive abnormalities in autotetraploid rice. Moreover, some meiosis-related genes were highly expressed in meiosis of both male and female meiocytes, while some showed reverse expression patterns [8]. We identified some special DEM associated with meiosis/meiosis-related genes by proteinprotein interaction networks. All of these interactions suggest that the DEM of MA and MM had a significant 
impact on the expressions of meiosis related or stagespecific genes. Additionally, these DEM showed opposite regulation between pollen and embryo sac, suggesting unsynchronized expression profiles in male and female gametophytes of autotetraploid rice. The regulation mechanisms of meiosis-related DEM were quite different between male and female gametophytes.

Earlier studies have shown that overexpression of miR159 inhibited mRNA levels of MYB family transcription factors leading to male sterility in Arabidopsis [20], and impairing the anthers development in rice [54]. In our study, osa-miR159a.1 targeted MYB family transcription factor $(O s G A M Y B$, responsible for defects in tapetal programmed cell death), which interacted with CYCA1;3, and displayed significant up-regulation in male meiosis but down-regulation in female meiosis. The increased expression of osa-miR159 during pollen development was consistent with a previous study on pollen sterile line [17]. We supposed that overexpression of osa-miR159a.1 in meiosis not only repressed the expression of MYB family transcription factor, but also affected the meiosis-related genes, which cause meiosis abnormalities and hampered normal pollen development in autotetraploid rice. In addition, osa-MIR159a-p5, another member of osa-miR159, targets LOC_Os09g32020 (ubiquitin fusion degradation protein, OsTKPR1), which displayed significant up-regulation in MA of autotetraploid rice. Ubiquitin fusion degradation protein families were important in the process of meiosis, and OsTKPR1 had reported to be associated with male sterility and pollen wall assembly [55, 56]. Highly-expressed osaMIR159a-p5 in MA may partially repressed the expression of LOC_Os09g32020 and cause meiosis abnormalities in 02428-4x.

osa-miR1436_L + 3_1ss5CT, targeted MCM9, which up-regulated in female meiosis of autotetraploid rice but down-regulated in male meiosis. Protein-protein interaction showed that MCM9 have a relationship with RPA2C (predicted by osa-miR167h-3p) and RPA2B, the genes related to meiosis. Replication protein A (RPA) is a single-stranded DNA binding protein that plays key role in DNA metabolic pathways. Incomplete meiotic chromosome fragmentation and synapsis were found in rpa1 mutant suggesting an essential role of RPA1 in DNA replication [57]. Additionally, recent research demonstrated a rice gene, OsMSH4/5 (Os07g0486000), which interacted with RPA complex and required for crossover formation during male and female gametes meiosis [40]. Here, up-regulation of osa-miR1436_L + 3_1ss5CT and osa-miR167h-3p in female meiosis might be negatively correlated with the expression of MCM9 and RPA2C, which displayed interaction with DNA replication genes, demonstrating that it may cause female meiosis abnormalities in autotetraploid rice.

\section{Down-regulation of $24 \mathrm{nt}$-phasiRNAs may cause} abnormalities during meiosis in autotetraploid rice $m i R 2118$ and $m i R 2275$ are required as triggers to initiate the $21 \mathrm{nt}$ and 24 nt-phasiRNAs biogenesis, and have been reported in rice and maize anther development [58-60]. MEL1, rice Argonaute protein, selectively binds the 21nt-phasiRNAs to play an important role in meiosis and male fertility [25]. Recently, Zhai et al. [26] analyzed the phasiRNAs of maize anther, and indicated that epidermis was necessary for producing $21 \mathrm{nt}$ pre-meiotic phasiRNA, whereas tapetal layer was required for $24 \mathrm{nt}$ meiotic phasiRNAs biogenesis; both of these phasiRNAs finally transport to meiotic cells and play their role in meiosis. Our previous study depicted different levels of expression of $m i R 2118$ and $m i R 2275$ families in Taichung65 [10]. In the present study, we found that $m i R 2118$ and miR2275 accumulated in pollen development and gradually declined from pre-meiotic, and 21 nt-phasiRNAs and 24 nt-phasiRNAs were also detected in the pollen development. The expression of miR2118 was hardly detected during embryo sac development; however, $21 \mathrm{nt}$-phasiRNAs not only detected in pollen development but also in the embryo sac development, suggesting that the $21 \mathrm{nt}$-phasiRNAs was not the anthers specific phasiRNAs; probably synthesized in other tissues then transported to embryo sac. Additionally, the irregular distribution of pollen-specific- 21 ntphasiRNAs in autotetraploid rice was different from the results of Zhai et al. [26], who indicated that the $21 \mathrm{nt}-$ phasiRNAs accumulated and peaked at pre-meiotic in maize. The expression patterns of 21 nt-phasiRNAs were more complicated in autotetraploid rice and remained unknown. Besides, the differentially expressed 24 nt-phasiRNAs were similar to the spatial-temporal expressions of miR2275d in 02428-4x pollen development. 24 ntphasiRNAs were absent in the aberrant meiocytes of maize [26]. The down-regulated $24 \mathrm{nt}$-phasiRNAs and miR2275d were associated with the high frequency of cytological abnormalities in 02428-4x. We speculated that down-regulation of miR2275 may result in the low expression of $24 \mathrm{nt}$-phasiRNAs, leading to the abnormal pollen mother cells development in autotetraploid rice.

\section{4 nt TEs-siRNAs may cause genome instability and infer-} tility in autotetraploid rice

The major function of $24 \mathrm{nt}$ TEs-siRNAs was to maintain the genome integrity by silencing transposable elements. Many transposable elements may activate in response to "genomic shock" by polyploidization of rice. In autotetraploid rice, DNA methylation variation of transposable elements was found to play crucial role to adapt the whole-genome duplication (WGD) against the polyploidization [28]. Most of the down-regulated $24 \mathrm{nt}$ siRNAs were associated with the accumulation of 
transcripts from DNA TEs in the mop1 mutant, which may relate with the loss of function of RNA-Dependent RNA Polymerase 2 (RDR2) [61]. Here, we identified various kinds of $24 \mathrm{nt}$ TEs-siRNAs associated with pollen and embryo sac development. In addition, the numbers of TEs-siRNAs were much higher during embryo sac development than pollen. The expression patterns of TEs-siRNAs were complex in different tissues of autotetraploid rice. Moreover, we found that $~ 50 \%$ TEs-siRNAs were differentially expressed in autotetraploid rice during pollen development and more than $80 \%$ of the differentially expressed TEs-siRNAs showed down-regulation, which indicated that reduction of $24 \mathrm{nt}$ TEs-siRNAs in pollen development of autotetraploid rice may induce genome instability and infertility during the pollen development. These results were consistent with the allopolyploid wheat and Arabidopsis, where down-regulated TEs-siRNAs contribute to genome destabilization [62, 63]. However, 24 nt TEssiRNAs were found to be up-regulated in embryo sac development. There was a great variation in the activity of RNA polymerase IV (Pol IV), DCL (Dicer-like) and Argonaute protein (AGO) in different development stage/tissue $[64,65]$, which resulted in differential expression patterns of TEs-siRNAs during pollen and embryo sac development. Moreover, siRNAs derived from transposable elements were similar to the nearby genes, which regulate the genes expression and play a crucial role in plant development $[66,67]$. Many genes expression levels may change under the influence of TEssiRNAs-triggered methylation, particularly at the crucial stages of female-meiosis, which explained more than 95\% up-regulated TEs-siRNAs during the embryo sac development in autotetraploid rice.

\section{Conclusion}

In the present study, we used high-throughput sequencing to identify the small RNA associated with the pollen and embryo sac development of autotetraploid and corresponding diploid rice. We identified 260 known and 488 novel miRNAs in autotetraploid and diploid rice, and 43 and 49 miRNAs specifically expressed during pollen and embryo sac development in autotetraploid rice, respectively. Various DEM were detected during pollen and embryo sac development in autotetraploid rice. Of these DEM, 18 and 21 important DEM were associated with male and female meiosis in autotetraploid rice, respectively. Moreover, abundantly expressed 21and 24-nt phasiRNAs exhibited differential expressions during pollen development, and 21-nt phasiRNAs were also detected during embryo sac development in the present study. Interestingly, up-regulated 24-nt TEssiRNAs were found to be dominant during the embryo sac development of autotetraploid rice, whereas a large proportion of down-regulated TEs-siRNAs were detected in pollen development. Consequently, the significant differential expression profiles of small RNAs in the autotetraploid rice may play important roles in regulating the development of pollen and embryo sac, thus resulting in low fertility and poor seed set in autotetraploid rice. Our findings provide a new insight on small RNA during pollen and embryo sac development in autotetraploid rice.

\section{Methods}

\section{Rice material}

Autotetraploid rice, 02428-4x, and its diploid rice (Oryza sativa L. subsp. japonica), 02428-2x, were used in this study. 02428-4x was developed from the chromosome doubling of $02428-2 x$ by colchicine and was self-crossed for more than 25 generations in our lab. They were planted at the experimental farm of South China Agricultural University (SCAU) under field conditions. Anthers with four pollen development stages, including pre-meiotic interphase (PMA), meiosis (MA), single microspore stage $(\mathrm{SCP})$ and bi-cellular pollen stage $(\mathrm{BCP})$, were collected from 02428-4x and 02428-2x based on the floret length and DAPI fluorescence (Additional file 4: Table S23). The ovary tissues were collected with their corresponding embryo sac development stages (i.e. megasporocyte formation stage (MF), megasporocyte meiosis stage (MM), functional megaspore formation stage (FMF) and eight-nucleate embryo sac developing-stage (EES)). All the samples were stored at $4{ }^{\circ} \mathrm{C}$ for cytological observation and at $-80{ }^{\circ} \mathrm{C}$ for RNA isolation.

\section{Analysis of agronomic/morphological traits}

A total of eleven morphological traits, including number of effective panicles, thousand kernel weight, plant height, flag leaf length, flag leaf width, panicle length, grain length, grain width, seed set, pollen and embryo sac fertility were examined to determine the phenotypic variations in autotetraploid and diploid rice.

\section{Cytological observation}

Anthers and ovaries were collected and fixed in Carnoy solution (ethanol: acetic acid $=3: 1 \mathrm{v} / \mathrm{v}$ ) for at least $24 \mathrm{~h}$. The samples were stored in $70 \%$ ethanol at $4{ }^{\circ} \mathrm{C}$ after washing with $95 \%$ ethanol for two times. Chromosome behavior and configurations were identified and analyzed by acetocarmine squashing as described by Wu et al. [6]. Meiosis stages were classified and explained according to $\mathrm{He}$ et al. [7]. To investigate the variation in pollen development of 02428-2x and 02428-4x, a whole mount eosin $B$ confocal laser scanning microscopy (WE-CLSM) was used. The samples were observed according to $\mathrm{Wu}$ et al. [6] with some minor modifications. The dissected anthers were hydrated in $50 \%, 30 \%$ ethanol and distilled water for 
5 min each. After an eosin B staining procedure for $30 \mathrm{~min}$, the samples were dehydrated in a series of ethanol solutions $(10 \%, 30 \%, 50 \%, 70 \%, 90 \%$ and $100 \%$ ethanol) for $5 \mathrm{~min}$. Finally, the dehydrated samples were transferred into a mixture of ethanol and methyl salicylate (1:1) for $1 \mathrm{~h}$, and then stored in pure methyl salicylate and observed under WE-CLSM. To investigate the variations in embryo sac development of 02428-2x and 02428-4x, WE-CLSM was used again and followed the procedure as described by Zeng et al. [68].

\section{Small RNA library construction and sequencing}

Total RNA was extracted from the anthers and ovaries by using Trizol reagent (Invitrogen, Carlsbad, CA, USA). Illumina's TruSeq small RNA sample preparation Kits (San Diego, CA, USA) were used to construct small RNA libraries. Then the small sequencing ( $36 \mathrm{bp}$ ) was performed on an Illumina Hiseq2500 at the LC-BIO (Hangzhou, China).

\section{Data processing/analysis of miRNAs}

After sequencing, the raw data was further processed to remove common RNA families (rRNA, tRNA, snRNA, snoRNA), repeats, low complexity, adapter dimers and junk by ACGT101-miR [10]. Then, the unique sequences (18-25 nt in length) were BLASTed to miRBase 20.0 (ftp://mirbase.org/pub/mirbase/) to identify known miRNAs. The novel miRNAs were predicted by using RNAfold software (http://rna.tbi.univie.ac.at/cgi-bin/RNAWebSuite/RNAfold.cgi). The miRNAs were considered as confidence miRNAs according to the normalized deep-sequencing levels (with the exclusion of $10 \mathrm{RPM}$ ) in all anthers and ovaries libraries. miRNAs with $P$-value (Fisher exact test and Chi-square $(X 2)$ test) $<0.05$ and $\mid \log 2$ (fold change ratio) $\mid>1$ were considered as differentially expressed miRNAs. Targeted genes of differentially expressed miRNAs were predicted by using TargetFinder [69]. GO enrichment analysis and Protein-protein interaction networks were performed using AgriGO (http:// bioinfo.cau.edu.cn/agriGO/) [70] and STRING (http:// string-db.org/) [71], respectively.

\section{Data processing/analysis of phasiRNAs}

We used PhaseTank to systemically characterize the 21 and 24 nt-phasiRNAs [41]. Then the confidence phasiRNAs with phasing scores higher than or equal to 25 were selected [26], and filtered the phasiRNAs that were triggered by miR2118 and miR2275 families. Finally, it yielded 1117 loci generating 21 nt-phasiRNAs and 190 loci generating 24 nt-phasiRNAs. PhasiRNAs with $P$-value (Fisher exact test) $<0.05$ and $\mid \log 2$ (fold change ratio) $\mid>1$ were considered as differentially expressed phasiRNAs.

\section{Data processing/analysis of TEs-siRNAs}

To find out the siRNAs associated with the transposable elements, we followed the method described by Zhang et al. [28]. Firstly, we filtered the reads that did not match miRNA, rRNA, tRNA, snRNA, or snoRNA, and then selected the reads which mapped to the transposable elements of rice reference genome. These subsequent of siRNAs were considered as TEs-siRNAs, and used for further analysis. The classifications of TEssiRNAs were done by $\mathrm{Li}$ et al. [10]. The differentially expressed TEs-siRNAs were identified by the conditions of $P$-value (Fisher exact test) $<0.05$ and $\mid \log 2$ (fold change ratio) $\mid>1$.

\section{Quantitative real-time PCR (qRT-PCR) analysis}

The total RNA from anthers and ovaries were used as template for reverse transcription with miRNA-specific stem-loop RT primers [72] by the Transcriptor First Strand cDNA Synthesis Kit (Roche). The reaction was as follow: incubated for $30 \mathrm{~min}$ at $16{ }^{\circ} \mathrm{C}$, followed by pulsed RT of 60 cycles at $30{ }^{\circ} \mathrm{C}$ for $30 \mathrm{~s}, 42{ }^{\circ} \mathrm{C}$ for $30 \mathrm{~s}$ and $50{ }^{\circ} \mathrm{C}$ for $1 \mathrm{~s}$, and a final incubation at $85{ }^{\circ} \mathrm{C}$ for $5 \mathrm{~min}$ to inactivate the reverse transcriptase [73]. The qRT-PCRs were performed on Lightcycler480 (Roche) using the SsoAdvanced Universal SYBR Green Supermix (Bio-RAD). The reaction conditions were as follows: $30 \mathrm{~s}$ at $95{ }^{\circ} \mathrm{C}, 40$ cycles of $95{ }^{\circ} \mathrm{C}$ denaturation for $5 \mathrm{~s}$ and $58{ }^{\circ} \mathrm{C}$ annealing and extension for $20 \mathrm{~s}$. Three biological replications were performed in this experiment. U6 snRNA was used as an internal reference for qRT-PCR of miRNAs. The relative expression of miRNAs was calculated by the $2^{-\triangle \Delta C T}$ method [74]. The stem-loop RT primers were designed by the Primer premier 5.0 software (Additional file 4: Table S24).

\section{Additional files}

\footnotetext{
Additional file 1: Figure S1. Chromosome observations of the diploid and autotetraploid rice. Figure S2. Cytological observation of pollen development in 02428-4x. Figure S3. Chromosome behavior during pollen mother cell (PMC) meiosis in 02428-4x. Figure S4. Cytological observation of embryo sac development in 02428-4x. Figure S5. Principa component analysis in each library. Figure S6. Validation of the miRNAs in 02428-4x and 02428-2x of pollen and embryo sac at each development stage. Figure S7. Venn analysis of the DEM (differentially expressed miRNAs) between pollen and embryo sac development in autotetraploid rice. Figure S8. Classification of the differentially expressed miRNAs between embryo sac and pollen development in autotetraploid rice. Figure S9. GO (Gene Ontology) enrichment analysis of predicted targets of DEM-P (pollenenriched miRNAs) in autotetraploid rice. Figure S10. GO enrichment analysis of predicted targets of DEM-ES (embryo sac-enriched miRNAs) in autotetraploid rice. Figure S11. GO enrichment analysis of predicted targets of differentially expressed miRNAs in MA (meiosis). Figure S12. GO enrichment analysis of predicted targets of differentially expressed miRNAs in MM (megasporocyte meiosis stage). Figure S13. Protein interactions between the targets of DEM (differentially expressed miRNAs) and meiosis-related genes. Figure S14. Distribution of TEs-siRNAs
} 
(siRNAs associated with transposable elements) in pollen and embryo sac development. (PDF $2706 \mathrm{~kb}$ )

Additional file 2: Table S1. Main agronomic traits of autotetraploid (02428-4x) and diploid (02428-2x) rice. Table S2. Frequencies of pollen fertility and embryo sac fertility in 02428-4x and 02428-2x. Table S3. The development of pollen and embryo sac in 02428-2x and 02428-4x. Table S4. Frequency of abnormalities in autotetraploid and diploid rice during the pollen development. Table S5. Observations of chromosome behavior and configurations between autotetraploid rice and diploid rice. Table S6. Summary of the known and predicted miRNAs in 02428-4x and 02428-2x. Table S7. miRNAs detected during pollen and embryo sac development of 02428-4x and 02428-2x. Table S8. The specific miRNAs found in each library (Development stages). (XLSX 214 kb)

Additional file 3: Table S9. Differentially expressed miRNAs (DEM) between diploid and autotetraploid during pollen development. Table S10. Differentially expressed miRNAs (DEM) between diploid and autotetraploid rice during embryo sac development. Table S11. Comparison of the differentially expressed miRNAs (DEM) between the pollen and embryo sac development in autotetraploid rice. Table S12. Differentially expressed miRNAs between embryo sac and pollen development in autotetraploid rice. Table S13. DEM-P (pollen-enriched miRNAs) and DEM-ES (embryo sac-enriched miRNAs) in 02428-4x. Table S14. Overall information about the predicted targets of detected miRNAs by Targetfinder. Table S15. Comparison of targets predicted by differentially expressed miRNAs with the previous studies on MA (meiosis). Table S16. Comparison of targets predicted by differentially expressed miRNAs with the previous studies on MM (megasporocyte meiosis stage). (XLSX $417 \mathrm{~kb}$ )

Additional file 4: Table S17. Protein-protein interaction of meiosisrelated genes with the targets predicted by the DEM associated with meiosis. Table S18. 21 nt-phasiRNAs triggered by the miR2118. Table S19. 24 nt-phasiRNAs triggered by the miR2275. Table S20. Overview of 24 nt TEs-siRNAs during pollen and embryo sac development of 02428-4x and 02428-2x. Table S21. Distribution of $24 \mathrm{nt}$ TEs-siRNAs in autotetraploid and diploid rice. Table S22. Differentially expressed 24 nt TEs-siRNAs during pollen and embryo sac development of autotetraploid rice. Table S23. Anther length during pollen development stages in autotetraploid and diploid rice. Table S24. The stem-loop RT primers used in the present study. (XLSX $1012 \mathrm{~kb}$ )

\section{Abbreviations}

BCP: (bi-cellular pollen stage); DEM: (differentially expressed miRNAs); EES: (eight-nucleate embryo sac developing-stage); EMC: Embryo sac mother cell; FMF: Functional megaspore formation stage; MA: Meiosis; MF: Megasporocyte formation stage; MM: Megasporocyte meiosis stage; PMA: Pre-meiotic interphase; PMC: Pollen mother cell; RPM: Reads per million; SCP: Single microspore stage

\section{Acknowledgements}

The authors thank Ms. Shuhong Yu and other lab members for assistance.

\section{Funding}

This work was supported by the NSFC (31571625 to XD Liu), Guangzhou Science and Technology Key Program (Application No. 201606201026123 to XD Liu) and the Guangdong Science and Technology Program (2014A030304055 to XD Liu).

\section{Availability of data and materials}

Small RNA sequencing raw data have been deposited to National Center for Biotechnology Information (NCBI), and accession number is "GSE90479".

\section{Authors' contributions}

$X D L$ and MQS conceived and designed the experiments. $X L, M Q S$ and $X D L$ wrote the paper. XL, MQS, JX, ZJL, NF, LW, JWW and ZXC performed the experiment and analyzed the data. All authors read and approved the final manuscript.

\section{Competing interests}

The authors declare that they have no competing interests.

\section{Consent for publication}

Not applicable.

\section{Ethics approval and consent to participate}

Not applicable.

Received: 23 November 2016 Accepted: 28 January 2017

Published online: 06 February 2017

\section{References}

1. Shahid MQ, Xu H, Lin S, Chen Z, Naeem M, Li Y, et al. Genetic analysis and hybrid vigor study of grain yield and other quantitative traits in autotetraploid rice. Pak J Bot. 2012;44:237-46.

2. Wu J, Hu C, Shahid MQ, Guo H, Zeng Y, Liu X, et al. Analysis on genetic diversification and heterosis in autotetraploid rice. Springerplus. 2013;2:439.

3. Shahid MQ, Li Y, Saleem MF, Naeem M, Wei C, Liu X. Yield and yield components in autotetraploid and diploid rice genotypes (indica and japonica) sown in early and late seasons. Aust J Crop Sci. 2013;7:632-41.

4. Guo H, Lu Y, Feng J, Yang B, Liu X. Further observation on the formation and development of autotetraploid rice embryo sac using laser scanning confocal microscopy. Acta Laser Biology Sinica. 2006;15:111-7.

5. Shahid MQ, Sun J, Wei C, Zhang P, Liu X. Studies on the abnormality of embryo sac and pollen fertility in autotetraploid rice during different growing seasons. Pak J Bot. 2010;42:7-19.

6. Wu J, Shahid MQ, Guo H, Yin W, Chen Z, Wang L, et al. Comparative cytological and transcriptomic analysis of pollen development in autotetraploid and diploid rice. Plant Reprod. 2014;27:181-96.

7. He J, Shahid MQ, Chen Z, Chen X, Liu X, Lu Y. Abnormal PMC microtubule distribution pattern and chromosome behavior resulted in low pollen fertility of an intersubspecific autotetraploid rice hybrid. Plant Syst Evol. 2011;291:257-65.

8. Kubo T, Fujita M, Takahashi H, Nakazono M, Tsutsumi N, Kurata N. Transcriptome analysis of developing ovules in rice isolated by laser microdissection. Plant Cell Physiol. 2013;54:750-65.

9. Wu Y, Yang L, Cao A, Wang J. Gene expression profiles in rice developing ovules provided evidence for the role of sporophytic tissue in female gametophyte development. PLoS One. 2015;10:e141613.

10. Li X, Shahid M, Wu J, Wang L, Liu X, Lu Y. Comparative small RNA analysis of pollen development in autotetraploid and diploid rice. Int J Mol Sci. 2016;17:499.

11. Boke H, Ozhuner E, Turktas M, Parmaksiz I, Ozcan S, Unver T. Regulation of the alkaloid biosynthesis by miRNA in opium poppy. Plant Biotechnol J. 2015;13:409-20.

12. Hong $Y$, Jackson S. Floral induction and flower formation-the role and potential applications of miRNAs. Plant Biotechnol J. 2015;13:282-92.

13. Shriram V, Kumar V, Devarumath RM, Khare TS, Wani SH. MicroRNAs as potential targets for abiotic stress tolerance in plants. Front Plant Sci. 2016;7:817.

14. Xie F, Jones DC, Wang Q, Sun R, Zhang B. Small RNA sequencing identifies miRNA roles in ovule and fibre development. Plant Biotechnol J. 2015;13:355-69.

15. Wei $L$, Yan $L$, Wang $T$. Deep sequencing on genome-wide scale reveals the unique composition and expression patterns of microRNAs in developing pollen of Oryza sativa. Genome Biol. 2011;12:R53.

16. Peng H, Chun J, Ai T, Tong $Y$, Zhang R, Zhao M, et al. MicroRNA profiles and their control of male gametophyte development in rice. Plant Mol Biol. 2012;80:85-102.

17. Yan J, Zhang $H$, Zheng $Y$, Ding $Y$. Comparative expression profiling of miRNAs between the cytoplasmic male sterile line MeixiangA and its maintainer line MeixiangB during rice anther development. Planta. 2015;241:109-23.

18. Peng T, Sun H, Du Y, Zhang J, Li J, Liu Y, et al. Characterization and expression patterns of microRNAs involved in rice grain filling. PLoS One. 2013;8:e54148.

19. Yi R, Zhu Z, Hu J, Qian Q, Dai J, Ding Y. Identification and expression analysis of microRNAs at the grain filling stage in rice (Oryza sativa L.) via deep sequencing. PLoS One. 2013;8:e57863.

20. Millar AA, Gubler F. The Arabidopsis GAMYB-like genes, MYB33 and MYB65, are microRNA-regulated genes that redundantly facilitate anther development. Plant Cell. 2005;17:705-21. 
21. Palatnik JF, Wollmann H, Schommer C, Schwab R, Boisbouvier J, Rodriguez R, et al. Sequence and expression differences underlie functional specialization of Arabidopsis microRNAs miR159 and miR319. Dev Cell. 2007;13:115-25.

22. Zhu Q, Upadhyaya NM, Gubler F, Helliwell CA. Over-expression of miR172 causes loss of spikelet determinacy and floral organ abnormalities in rice (Oryza sativa). BMC Plant Biol. 2009;9:149.

23. Zhang Y, Yu Y, Wang C, Li Z, Liu Q, Xu J, et al. Overexpression of microRNA OsmiR397 improves rice yield by increasing grain size and promoting panicle branching. Nat Biotechnol. 2013;31:848-52.

24. Wu MF, Tian Q, Reed JW. Arabidopsis microRNA167 controls patterns of ARF6 and ARF8 expression, and regulates both female and male reproduction. Development. 2006;133:4211-8.

25. Komiya R, Ohyanagi H, Niihama M, Watanabe T, Nakano M, Kurata N, et al. Rice germline-specific Argonaute MEL1 protein binds to phasiRNAs generated from more than 700 lincRNAs. Plant J. 2014;78:385-97.

26. Zhai J, Zhang H, Arikit S, Huang K, Nan G, Walbot V, et al. Spatiotemporally dynamic, cell-type-dependent premeiotic and meiotic phasiRNAs in maize anthers. Proc Natl Acad Sci USA. 2015;112:3146-51.

27. Chen C, Farmer AD, Langley RJ, Mudge J, Crow JA, May GD, et al. Meiosisspecific gene discovery in plants: RNA-Seq applied to isolated Arabidopsis male meiocytes. BMC Plant Biol. 2010;10:280.

28. Zhang J, Liu Y, Xia E, Yao Q, Liu X, Gao L. Autotetraploid rice methylome analysis reveals methylation variation of transposable elements and their effects on gene expression. Proc Natl Acad Sci USA. 2015;112:E7022-9.

29. Fujita M, Horiuchi Y, Ueda Y, Mizuta Y, Kubo T, Yano K, et al. Rice expression atlas in reproductive development. Plant Cell Physiol. 2010;51:2060-81.

30. Deveshwar P, Bovill WD, Sharma R, Able JA, Kapoor S. Analysis of anther transcriptomes to identify genes contributing to meiosis and male gametophyte development in rice. BMC Plant Biol. 2011;11:78.

31. Jin $Y$, Yang $H$, Wei Z, Ma H, Ge X. Rice male development under drought stress: phenotypic changes and stage-dependent transcriptomic reprogramming. Mol Plant. 2013;6:1630-45.

32. Aya K, Ueguchi-Tanaka M, Kondo M, Hamada K, Yano K, Nishimura M, et al. Gibberellin modulates anther development in rice via the transcriptional regulation of GAMYB. Plant Cell. 2009;21:1453-72.

33. Wu J, Shahid MQ, Chen L, Chen Z, Wang L, Liu X, et al. Polyploidy enhances $F_{1}$ pollen sterility loci interactions that increase meiosis abnormalities and pollen sterility in autotetraploid rice. Plant Physiol. 2015;169:2700-17.

34. Wei LQ, Xu WY, Deng ZY, Su Z, Xue Y, Wang T. Genome-scale analysis and comparison of gene expression profiles in developing and germinated pollen in Oryza sativa. BMC Genomics. 2010;11:338.

35. Honys D, Twell D. Transcriptome analysis of haploid male gametophyte development in Arabidopsis. Genome Biol. 2004;5:R85.

36. Yant L, Hollister JD, Wright KM, Arnold BJ, Higgins JD, Franklin FC, et al. Meiotic adaptation to genome duplication in Arabidopsis arenosa. Curr Biol. 2013;23:2151-6.

37. Wright KM, Arnold B, Xue K, Urinova M, O'Connell J, Bomblies K. Selection on meiosis genes in diploid and tetraploid Arabidopsis arenosa. Mol Biol Evol. 2015;32:944-55.

38. Ohnishi T, Takanashi H, Mogi M, Takahashi H, Kikuchi S, Yano K, et al. Distinct gene expression profiles in egg and synergid cells of rice as revealed by cell type-specific microarrays. Plant Physiol. 2011;155:881-91.

39. Schmidt A, Wuest SE, Vijverberg K, Baroux C, Kleen D, Grossniklaus U. Transcriptome analysis of the Arabidopsis megaspore mother cell uncovers the importance of RNA helicases for plant germline development. PLoS Biol. 2011;9:e1001155

40. Wang C, Wang Y, Cheng Z, Zhao Z, Chen J, Sheng P, et al. The role of OsMSH4 in male and female gamete development in rice meiosis. J Exp Bot. 2016;67:1447-59.

41. Guo Q, Qu X, Jin W. PhaseTank: genome-wide computational identification of phasiRNAs and their regulatory cascades. Bioinformatics. 2015;31:284-6.

42. Fan G, Zhai X, Niu S, Ren Y. Dynamic expression of novel and conserved microRNAs and their targets in diploid and tetraploid of Paulownia tomentosa. Biochimie. 2014;102:68-77.

43. Niu S, Fan G, Xu E, Deng M, Zhao Z, Dong Y. Transcriptome/Degradomewide discovery of microRNAs and transcript targets in two Paulownia australis genotypes. PLoS One. 2014;9:e106736.

44. Niu S, Fan G, Zhao Z, Deng M, Dong Y. High-throughput sequencing and degradome analysis reveal microRNA differential expression profiles and their targets in Paulownia fortunei. Plant Cell Tiss Org. 2014;119: 457-68.
45. Zhai X, Niu S, Ren Y, Fan G. Discovery and profiling of microRNAs and their targets in Paulownia 'Yuza 1' plants via high-throughput sequencing and degradome analysis. Genes Genom. 2016;38:757-66.

46. Chagué V, Just J, Mestiri I, Balzergue S, Tanguy AM, Huneau C, et al. Genome-wide gene expression changes in genetically stable synthetic and natural wheat allohexaploids. New Phytol. 2010;187:1181-94.

47. Chelaifa H, Chagué V, Chalabi S, Mestiri I, Arnaud D, Deffains D, et al. Prevalence of gene expression additivity in genetically stable wheat allohexaploids. New Phytol. 2013;197:730-6.

48. Yoo MJ, Szadkowski E, Wendel JF. Homoeolog expression bias and expression level dominance in allopolyploid cotton. Heredity. 2013;110:171-80.

49. Li X. Natural attributes and agricultural implications of somatic genome variation. Curr Issues Mol Biol. 2016;20:29-46.

50. Nazzicari N, Biscarini F, Cozzi P, Brummer EC, Annicchiarico P. Marker imputation efficiency for genotyping-by-sequencing data in rice (Oryza sativa) and alfalfa (Medicago sativa). Mol Breeding. 2016;36:69.

51. Ha M, Lu J, Tian L, Ramachandran V, Kasschau KD, Chapman EJ, et al. Small RNAs serve as a genetic buffer against genomic shock in Arabidopsis interspecific hybrids and allopolyploids. Proc Natl Acad Sci USA. 2009;106:17835-40.

52. Xie F, Zhang B. microRNA evolution and expression analysis in polyploidized cotton genome. Plant Biotechnol J. 2015;13:421-34.

53. Zhang $H$, Zhao H, Wu S, Huang F, Wu K, Zeng $X$, et al. Global methylation patterns and their relationship with gene expression and small RNA in rice lines with different ploidy. Front Plant Sci. 2016;7:1002.

54. Tsuji H, Aya K, Ueguchi-Tanaka M, Shimada Y, Nakazono M, Watanabe R, et al. GAMYB controls different sets of genes and is differentially regulated by microRNA in aleurone cells and anthers. Plant J. 2006;47:427-44.

55. Quilichini TD, Grienenberger E, Douglas CJ. The biosynthesis, composition and assembly of the outer pollen wall: A tough case to crack. Phytochemistry. 2015;113:170-82.

56. Wang Y, Lin YC, So J, Du Y, Lo C. Conserved metabolic steps for sporopollenin precursor formation in tobacco and rice. Physiol Plant. 2013:149:13-24.

57. Aklilu BB, Soderquist RS, Culligan KM. Genetic analysis of the Replication Protein A large subunit family in Arabidopsis reveals unique and overlapping roles in DNA repair, meiosis and DNA replication. Nucleic Acids Res. 2014;42: 3104-18.

58. Johnson C, Kasprzewska A, Tennessen K, Fernandes J, Nan GL, Walbot V, et al. Clusters and superclusters of phased small RNAs in the developing inflorescence of rice. Genome Res. 2009;19:1429-40.

59. Zhai J, Jeong DH, De Paoli E, Park S, Rosen BD, Li Y, et al. MicroRNAs as master regulators of the plant NB-LRR defense gene family via the production of phased, trans-acting siRNAs. Genes Dev. 2011;25:2540-53.

60. Song X, Li P, Zhai J, Zhou M, Ma L, Liu B, et al. Roles of DCL4 and DCL3b in rice phased small RNA biogenesis. Plant J. 2012;69:462-74.

61. Jia Y, Lisch DR, Ohtsu K, Scanlon MJ, Nettleton D, Schnable PS. Loss of RNAdependent RNA polymerase 2 (RDR2) function causes widespread and unexpected changes in the expression of transposons, genes, and 24-nt small RNAs. PLoS Genet. 2009;5:e1000737.

62. Kenan-Eichler M, Leshkowitz D, Tal L, Noor E, Melamed-Bessudo C, Feldman $M$, et al. Wheat hybridization and polyploidization results in deregulation of small RNAs. Genetics. 2011;188:263-72.

63. Slotkin RK, Vaughn M, Borges F, Tanurdzic M, Becker JD, Feijo JA, et al. Epigenetic reprogramming and small RNA silencing of transposable elements in pollen. Cell. 2009;136:461-72.

64. Mosher RA, Melnyk CW, Kelly KA, Dunn RM, Studholme DJ, Baulcombe DC. Uniparental expression of PollV-dependent siRNAs in developing endosperm of Arabidopsis. Nature. 2009;460:283-6.

65. Wierzbicki AT, Haag JR, Pikaard CS. Noncoding transcription by RNA polymerase Pol IVb/Pol V mediates transcriptional silencing of overlapping and adjacent genes. Cell. 2008;135:635-48.

66. Liu J, He Y, Amasino R, Chen X. siRNAs targeting an intronic transposon in the regulation of natural flowering behavior in Arabidopsis. Genes Dev. 2004;18:2873-8.

67. Henderson IR, Jacobsen SE. Tandem repeats upstream of the Arabidopsis endogene SDC recruit non-CG DNA methylation and initiate siRNA spreading. Genes Dev. 2008;22:1597-606.

68. Zeng Y, Hu C, Lu Y, Li J, Liu X. Diversity of abnormal embryo sacs in indical japonica hybrids in rice demonstrated by confocal microscopy of ovaries. Plant Breeding. 2007;126:574-80. 
69. Fahlgren N, Carrington JC. miRNA target prediction in plants. Methods Mol. Biol. 2010;592:51-7.

70. Du Z, Zhou X, Ling Y, Zhang Z, Su Z. agriGO: a GO analysis toolkit for the agricultural community. Nucleic Acids Res. 2010;38:W64-70,

71. Szklarczyk D, Franceschini A, Wyder S, Forslund K, Heller D, Huerta-Cepas J, et al. STRING v10: protein-protein interaction networks, integrated over the tree of life. Nucleic Acids Res. 2015;43:D447-52.

72. Chen C, Ridzon DA, Broomer AJ, Zhou Z, Lee DH, Nguyen JT, et al. Realtime quantification of microRNAs by stem-loop RT-PCR. Nucleic Acids Res. 2005;33:e179.

73. Varkonyi-Gasic E, Wu R, Wood M, Walton EF, Hellens RP. Protocol: a highly sensitive RT-PCR method for detection and quantification of microRNAs. Plant Methods. 2007;3:12

74. Livak KJ, Schmittgen TD. Analysis of relative gene expression data using real-time quantitative PCR and the $2^{-\Delta \Lambda C T}$ Method. Methods. 2001;25:402-8.

\section{Submit your next manuscript to BioMed Central} and we will help you at every step:

- We accept pre-submission inquiries

- Our selector tool helps you to find the most relevant journal

- We provide round the clock customer support

- Convenient online submission

- Thorough peer review

- Inclusion in PubMed and all major indexing services

- Maximum visibility for your research

Submit your manuscript at www.biomedcentral.com/submit 